\title{
Constant-Envelope Space-Time Shift Keying
}

\author{
Chao Xu, Member, IEEE, Tong Bai, Student Member, IEEE, Jiankang Zhang, Senior Member, IEEE, Robert G. \\ Maunder, Senior Member, IEEE, Shinya Sugiura, Senior Member, IEEE, Zhaocheng Wang, Senior Member, IEEE, \\ and Lajos Hanzo* Fellow, IEEE
}

\begin{abstract}
From the power amplifier's perspective, the Peak-toAverage Power Ratio (PAPR) is of essential importance, especially for both single-RF and reduced-RF Multiple-Input MultipleOutput (MIMO) single-carrier schemes. In this context, many of the diversity-oriented index modulation schemes - including the full-RF Space-Time Shift Keying (STSK) and the single-RF Asynchronous STSK (ASTSK) that invoke randomized signals - exhibit eroded energy-efficiency. To circumvent this problem, we propose a holistic signal construction approach for single-RF, reduced-RF and full-RF MIMO setups, which always achieve both perfect 0 dB PAPR transmission and Inter-Channel Interference (ICI) free signal detection. More explicitly, first of all, we conceive a new family of single-RF Constant-Envelope ASTSK (CE-ASTSK), which is capable of substantially outperforming conventional Spatial Modulation (SM) in both Rayleigh fading and Ricean fading associated with increasing Line-of-Sight (LoS) power. Secondly, we propose the new full-RF CE-STSK concept, which is capable of outperforming the orthogonal SpaceTime Block Codes (STBCs) without either increasing PAPR or imposing ICI. This is particularly beneficial because the conventional Linear Dispersion Code (LDC) approaches always compromise the orthogonality of STBC and hence impose ICI. Thirdly, we also conceive the reduced-RF versions of CE-STSK, which outperform both Generalized Spatial Modulation (GSM) and Space-Time Block Coded Spatial Modulation (STBC-SM). Finally, the proposed schemes are intrinsically amalgamated with turbo detection assisted channel coding, which further confirms the superiority of CE-ASTSK and CE-STSK over SM and STBC in the single-RF and full-RF modes, respectively.
\end{abstract}

Index Terms-Index modulation, spatial modulation, spacetime shift keying, constant-envelope, peak-to-average power ratio, inter-channel-interference, Ricean fading, turbo detection.

\section{INTRODUCTION}

In recent years, the Index Modulation (IM) philosophy has been applied to a variety of single-RF and reducedRF Multiple-Input Multiple-Output (MIMO) schemes [1][10], which aim for reducing the power consumption at the transmitter by activating a reduced number of RF chains. As one of the most power-thirsty RF components, the critical goal of Power-Amplifier (PA) design is to reduce the Peakto-Average Power Ratio (PAPR) [11]-[14], as portrayed in

C. Xu, J. Zhang, R. G. Maunder and L. Hanzo are with the School of Electronics and Computer Science, University of Southampton, Southampton SO17 1BJ, UK (e-mail: \{cx1g08,jz09v,rm,lh\}@soton.ac.uk). T. Bai is with the School of Electronic Engineering and Computer Science, Queen Mary University of London, London E1 4NS, UK (email: t.bai@qmul.ac.uk). S. Sugiura is with the Institute of Industrial Science, University of Tokyo, Meguro-ku, Tokyo 153-8505, Japan (e-mail: sugiura@ieee.org). Z. Wang is with Tsinghua University, Beijing, China (e-mail: zcwang@tsinghua.edu.cn).

L. Hanzo would like to acknowledge the financial support of the Engineering and Physical Sciences Research Council projects EP/Noo4558/1, EP/PO34284/1, COALESCE, of the Royal Societys Global Challenges Research Fund Grant, of the Royal Society Grant IF170002 as well as of the European Research Councils Advanced Fellow Grant QuantCom. The work of S. Sugiura was supported in part by the Japan Society for the Promotion of Science KAKENHI under Grants 26709028 and 16KK0120.

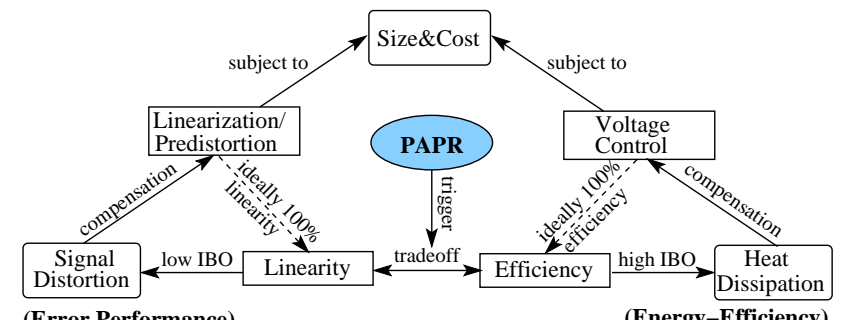

Error Performance)

(Energy-Efficiency)

Fig. 1: Triangular tradeoffs in Power Amplifier (PA) design.

Fig. 1. The Input Back-Off (IBO) seen in Fig. 1 is defined by the ratio of maximum voltage supply and average input power. For improving the linearity, a high IBO is required for mitigating the signal distortion. However, the high-PAPR signal associated with a fluctuating envelope only occasionally has a peak voltage, yet perpetually degrading the PA efficiency, as indicated in Fig. 1. As a remedy, on one hand, the classic analog feedback/feedforward linearization as well as the digital predistortion technique of Fig. 1 may be invoked for restoring the ideal linearity in the face of low IBO. Nonetheless, the performance of the analog linearization circuits is dependent on the operating frequency, while sophisticated digital predistortion is only suitable for the Base Station (BS) [12]. On the other hand, in order to mitigate the heat dissipation from using high IBO, we may arrange for adaptively controlling the supply voltage according to the signal envolope. For instance, the classic Doherty technique is constituted by a main class-B PA and an auxiliary class-C PA, where the latter is only switched on for high signal amplitudes. Even though the transistors can be turned on and off at an ultrasonic rate [15], the maximum achievable efficiency of commercially available PAs remains limited to $20 \%-35 \%$ [12], [13], which indicates a substantial heat dissipation, regardless of the number of activated RF chains.

From the PA's perspective, there is an energy-efficiency tradeoff between single-RF, reduced-RF and full-RF MIMO setups, which is exemplied and summarized in Fig. 2. On one hand, it is widely acknowledged that even at a zero PA output power of $P_{\text {out }}=0$, the transmitter architecture still consumes non-negligible power, which may even be as high as $50 \%$ of the PA's input power $P_{i n}$ [16]. The associated Transmit Power Independent (TPI) term in Fig. 2 includes the RF-drive power as well as an active cooling system [12]. Naturally, this TPI grows linearly with the number of activated RF chains $M_{A}$, as seen in Fig. 2. On the other hand, the PA of the single-RF system has to deliver $M$ times higher output power $P_{\text {out }}=P_{t}$ than its counterparts operating in the fullRF mode associated with $P_{\text {out }}=P_{t} / M$, where $M$ denotes the number of Transmit Antennas (TAs), while $P_{t}$ denotes the 


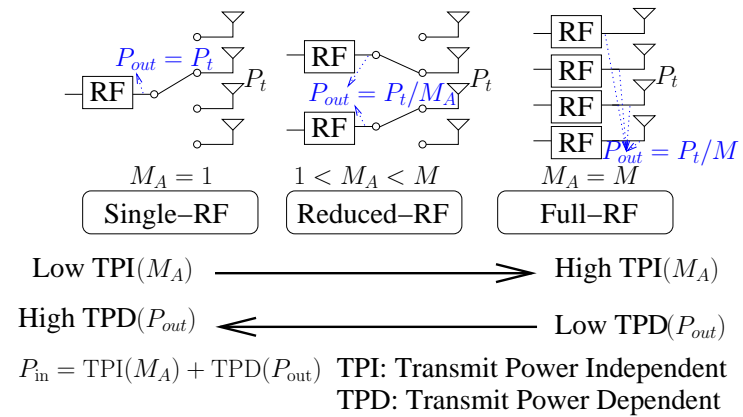

Fig. 2: The tradeoff between single-RF, reduced-RF and full-RF schemes from the PA's perspective.

total radiation power [17]. As a result, the Transmit Power Dependent (TPD) term, which has been shown empirically to be a linear function of $P_{\text {out }}$ [12], exhibits a reversed trend with $M_{A}$ in Fig. 2. In summary, the reduction in the number of RF chains does not automatically lead to a reduced overall PA power consumption $P_{i n}$. A reasonable rule of thumb is that when a lower-gain PA is employed, the RF-drive power is of a higher proportion in $P_{i n}$ [11], hence reducing the number of RF chains may become more beneficial. The low-gain PAs are particularly popular for the power-hungry devices both in the next-generation terrestrial uplink and in Air-to-Ground (A2G) communication systems.

Moreover, since the multi-carrier Orthogonal FrequencyDivision Multiplexing (OFDM) activates all the RF chains, the family of single-RF and reduced-RF MIMO schemes tend to rely on single-carrier transmission [2]-[4], where frequency-domain equalization may be invoked both at the terrestrial BS and at the aerial Ground Station (GS). In these scenarios, typically Ricean fading associated with increased Line-of-Sight (LoS) power is encountered. The resultant antenna correlation is especially detrimental to single-RF Spatial Modulation (SM) [5], owing to the fact that SM relies on the independently fading channel coefficients to convey the IM bits. As a rememdy, transmit diversity may be introduced. It was demonstrated in [18], [19] that transmit diversity is especially robust against the grave airframe-induced fading, where the critical A2G link may become blocked by the fuselage of Unmanned Aerial Vehicle (UAV). However, the state-of-the-art diversity-oriented IM schemes including both the full-RF Space-Time Shift Keying (STSK) [20], [21], as well as the single-RF Asynchronous STSK (ASTSK) [20], [22] and the SM using Complex Interleaved Orthogonal Design (SM-CIOD) [23] are not sufficiently energy-efficient due to their high-PAPR. Against this background, in this work, we have conceived a holistic signal construction approach that pursues the following imperative objectives for all the singleRF, reduced-RF and full-RF MIMO setups:

(I) Low-Complexity Transmitter: The Constant-Envolope (CE) design ensures having a perfect $0 \mathrm{~dB}$ PAPR for signal transmission.

(II) Low-Complexity Receiver: The STSK structure offers Inter-Channel Interference (ICI)-free signal detection.

(III) Diversity Gain: The space-time optimization of the STSK's dispersion matrix maximizes the diversity gain.

These beneficial qualities of the proposed schemes are
TABLE I: Summary of MIMO schemes.

\begin{tabular}{|c|c|c|c|c|c|}
\hline \multicolumn{2}{|c|}{ MIMO Scheme } & $\begin{array}{l}\text { PAPR } \\
\text { (Transmitter) }\end{array}$ & \begin{tabular}{l|l} 
ICI \\
(Receiver)
\end{tabular} & $\begin{array}{l}\text { Transmit } \\
\text { Diversity }\end{array}$ & $\begin{array}{l}\text { Transmission } \\
\text { Delay }\end{array}$ \\
\hline \multirow{5}{*}{$\begin{array}{l}\text { Single-RF } \\
\left(M_{A}=1\right)\end{array}$} & SM [5] & $\begin{array}{l}\text { PAPR }=0 \mathrm{~dB} \\
(\mathrm{PSK})\end{array}$ & $\mathrm{ICI}=0$ & $\times$ & $T=1$ \\
\hline & SM-CIOD [23] & High PAPR & $\mathrm{ICI}=0$ & $\sqrt{ }$ & $T=2$ \\
\hline & ASTSK [20], [22] & High PAPR & $\mathrm{ICI}=0$ & $\sqrt{ }$ & $T=M$ \\
\hline & $\begin{array}{l}\text { CE-ASTSK } \\
\text { (TAST/PAST) }\end{array}$ & $\mathrm{PAPR}=0 \mathrm{~dB}$ & $\mathrm{ICI}=0$ & $\sqrt{ }$ & $T=M$ \\
\hline & $\begin{array}{l}\text { CE-ASTSK-DR } \\
\text { (Type I/Type II) }\end{array}$ & $\mathrm{PAPR}=0 \mathrm{~dB}$ & $\mathrm{ICI}=0$ & $\sqrt{ }$ & $T=\frac{M}{W}$ \\
\hline \multirow{7}{*}{$\begin{array}{l}\text { Full-RF } \\
\left(M_{A}=M\right)\end{array}$} & V-BLAST [24] & $\begin{array}{l}\text { PAPR }=0 \mathrm{~dB} \\
(\mathrm{PSK})\end{array}$ & $\mathrm{ICI}=M$ & $\times$ & $T=1$ \\
\hline & STBC [25]-[28] & $\begin{array}{l}\text { PAPR }=0 \mathrm{~dB} \\
(\mathrm{PSK})\end{array}$ & $\mathrm{ICI}=0$ & $\sqrt{ }$ & $T \geq M$ \\
\hline & $\begin{array}{l}\text { QO-STBC [29], } \\
{[30]}\end{array}$ & $\begin{array}{l}\mathrm{PAPR}=0 \mathrm{~dB} \\
(\mathrm{PSK})\end{array}$ & $\mathrm{ICI}=2$ & $\sqrt{ }$ & $T=M$ \\
\hline & LDC [31]-[37] & High PAPR & $\mathrm{ICI}=Q$ & $\sqrt{ }$ & $T=M$ \\
\hline & STSK $[20],[21]$ & High PAPR & $\mathrm{ICI}=0$ & $\sqrt{ }$ & $T=M$ \\
\hline & $\begin{array}{l}\text { CE-STSK } \\
\text { (TAST/PAST) }\end{array}$ & $\mathrm{PAPR}=0 \mathrm{~dB}$ & $\mathrm{ICI}=0$ & $\sqrt{ }$ & $T=M$ \\
\hline & $\begin{array}{l}\text { CE-STSK-DR } \\
\text { (Type I/Type II) }\end{array}$ & $\mathrm{PAPR}=0 \mathrm{~dB}$ & $\mathrm{ICI}=0$ & $\sqrt{ }$ & $T=\frac{M}{W}$ \\
\hline \multirow{4}{*}{$\begin{array}{l}\text { Reduced-RF } \\
\left(1<M_{A}<M\right)\end{array}$} & GSM [7]-[9] & $\begin{array}{l}\text { PAPR }=0 \mathrm{~dB} \\
(\mathrm{PSK})\end{array}$ & $\begin{array}{c}\mathrm{ICI} \\
M_{A}\end{array}$ & $\times$ & $T=1$ \\
\hline & $\begin{array}{ll}\text { STBC-SM } & {[6],} \\
{[38]} & \\
\end{array}$ & $\begin{array}{l}\mathrm{PAPR}=0 \mathrm{~dB} \\
(\mathrm{PSK})\end{array}$ & $\begin{array}{l}\mathrm{ICI} \\
M_{A}\end{array}$ & $\sqrt{ }$ & $T=M_{A}$ \\
\hline & $\begin{array}{l}\text { CE-STSK-RH } \\
\text { (Type I/Type II) }\end{array}$ & $\mathrm{PAPR}=0 \mathrm{~dB}$ & $\mathrm{ICI}=0$ & $\sqrt{ }$ & $T=M_{A}$ \\
\hline & CE-GSTSK & $\mathrm{PAPR}=0 \mathrm{~dB}$ & $\begin{array}{l}\mathrm{ICI} \\
M_{A}\end{array}$ & $\sqrt{ }$ & $T=\frac{M}{M_{A}}$ \\
\hline
\end{tabular}

highlighted in Table I in comparison to the existing MIMO schemes. More explicitly, our novel contributions are:

(1) First of all, we conceive the single-RF CE-ASTSK scheme using both the Threaded Algebraic Space-Time (TAST) [33], [39] and the new Permuted Algebraic Space-Time (PAST) signal structures. More explicitly, in the $(T \times T)$-element signal matrix, the CE-ASTSK using TAST activates a single one out of $T$ non-overlapping signal positions, where a single LPSK symbol is dispersed by the activated signal thread. In contrast to both the conventional TAST of [33], [39] and to the ASTSK of [20], [22], the proposed CE-ASTSK transmits at $\mathrm{PAPR}=0 \mathrm{~dB}$ and receives free from ICI. Moreover, instead of switching between $T$ threads, the new PAST scheme allows full permutation of all signal positions, which hence conveys a higher number of $\left\lfloor\log _{2} T !\right\rfloor$ IM bits. The price paid is that the overlapping signal positions have to be assigned with additional phase rotations.

(2) As the number of TAs $M$ grows, it becomes desirable to limit the number of transmission time slots $T$. For this reason, we devise two types of Diversity-Rate (DR) tradeoff arrangements, which are termed as the Type I and Type II CE-ASTSK-DR schemes in Table I. More explicitly, the Type I of CE-ASTSK-DR partitions the $(T \times M)$-element signal matrix into $W=M / T$ number of submatrices, where a single one out of $W$ is activated for transmitting a $(T \times T)$-element sparse TAST signal matrix. As a result, on one hand, the signal construction is simplified for large $M$. On the other hand, an extra number of $\left\lfloor\log _{2} W\right\rfloor$ IM bits are conveyed. In order to improve the throughput, the Type II CE-ASTSK-DR scheme allows the full set of $T$ out of $M$ signal position combinations, which hence conveys an increased number of $\left\lfloor\log _{2}\left(\begin{array}{c}M \\ T\end{array}\right)\right\rfloor$ IM bits.

(3) In order to offer a holistic design, we propose to trans- 
form the single-RF CE-ASTSK and the CE-ASTSK-DR to the full-RF CE-STSK and CE-STSK-DR schemes, respectively, as seen in Table I. This is achieved upon multiplying their signal matrix by a normalized $(M \times$ $M$ )-element Hadamard matrix, which neither increases PAPR nor imposes ICI. To elaborate, the Hadamard matrix was originally invoked in [39] for the sake of balancing the average transmit power. However, without amalgamating it with the IM philosophy, the employment of Hadamard matrix on its own fails to guarantee PAPR $=0 \mathrm{~dB}$ transmission and ICI-free reception. As a result, the proposed full-RF CE-STSK and CE-STSKDR become capable of outperforming both Alamouti's G2 Space-Time Block Code (STBC) [25] as well as the 1/2-rate and 3/4-rate orthogonal STBCs [26]-[28]. This is a significant novelty compared to the conventional approach of improving the STBC performance at the cost of imposing ICI, which includes the Quasi-Orthogonal (QO) STBCs [29], [30] as well as the Linear Dispersion Code (LDC) solutions [31]-[37] seen in Table I.

(4) As a further advance, we also conceive the reduced-RF versions of CE-STSK, which are capable of outperforming both Generalized Spatial Modulation (GSM) [7][9] and Space-Time Block Coded Spatial Modulation (STBC-SM) [6], [38]. When $M_{A}=T$ out of $M$ TAs are activated, we multiply the sparse signal matrix by a normalized $\left(M_{A} \times M_{A}\right)$-element Reduced-size Hadamard (RH) matrix, resulting in the CE-STSK-RH scheme of Table I. In order to improve the throughput, we further propose a CE Generalized STSK (CE-GSTSK) solution, which achieves substantial performance improvements over GSM associated with the same ICI of $M_{A}$ streams.

(5) Finally, the proposed CE-ASTSK and CE-STSK are amalgamated with the classic Low-Density Parity-Check (LDPC) coding scheme. We invoke EXtrinsic Information Transfer (EXIT) charts [40]-[43] for analysing the convergence behaviour of the turbo detection assisted receiver. Our simulation results confirm the impressive advantages of CE-ASTSK and CE-STSK over SM and STBC in the single-RF and full-RF modes, respectively.

We explicitly note that the TAST conceived for CE-ASTSK has been substantially improved compared to our previous finite-cardinality Differential STSK (DSTSK) of [44]. In the absence of differential encoding, we have dispensed with the restrictions of $T \geq M$ and $L_{D M}=2^{\iota}$ for an integer $\iota$ in [44], and the corresponding methodology of the dispersion matrix optimization is reformulated. As a result, we devise a wide-range of high-throughput STSK arrangements, as seen in Table I, where opting for $T \leq M$ is the key to both our simplified design and to its improved spectral efficiency. Moreover, we have recently developed a family of GSTSK schemes that use both $0 \mathrm{~dB}$ PAPR PSK and low-PAPR star QAM in [45]. More explicitly, the GSTSK arrangements of [45] are associated with ICI $=M$ and they outperform the full-diveristy full-rate MIMO schemes of perfect STBCs [33], [34], [36], [37] including the Golden code [35], both of which suffer from a high PAPR and from an increased ICI $=M^{2}$.
By contrast, the family of ICI-free STSK schemes proposed in this work aim for outperforming the ICI-free SM and STBC schemes. The only exception is constituted by the CESTSK-RH scheme seen in Table I, which still has a reduced $\mathrm{ICI}=M_{A}<M$.

In the rest of this paper, the system overview is presented in Sec. II. The single-RF CE-ASTSK schemes are developed in Sec. III, while its full-RF and reduced-RF CE-STSK counterparts are conceived in Sec. IV. The performance results are offered in Sec. V. Our turbo detection assisted LDPC coded system is presented in Sec. VI, while our conclusions are offered in Sec. VII.

\section{SYSTEM OVERVIEW}

\section{A. Channel Model}

The signals received at $N$ Receive Antennas (RAs) over $T$ time slots are:

$$
\mathbf{Y}_{n}=\mathbf{S}_{n} \mathbf{H}_{n}+\mathbf{V}_{n}
$$

where the $(T \times M)$-element matrix $\mathbf{S}_{n}$ represents the signals transmitted from $M$ TAs, while the $(T \times N)$-element $\mathbf{Y}_{n}$ and $\mathbf{V}_{n}$ model the received signals and the AWGN, respectively. The Ricean fading channels are modelled as [46]-[49]:

$$
\mathbf{H}_{n}=\left(\mathbf{H}_{n}^{D}+\mathbf{H}_{n}^{S}\right) e^{j \theta} .
$$

The $(M \times N)$-element LOS channel-matrix is given by $\mathbf{H}_{n}^{D}=\sigma_{D} e^{j 2 \pi \Delta f_{\operatorname{LoS} n}} \mathbf{a}_{t} \mathbf{a}_{r}^{T}$, where $\sigma_{D}^{2}$ denotes the LOS power. The LOS frequency offset is $\Delta f_{\mathrm{LOS}}=f_{d} \cos \left(\phi_{0}\right) \leq$ $f_{d}$, where $f_{d}$ refers to the normalized maximum Doppler frequency, while $\phi_{0}$ is the angle between the LOS and the moving direction. The signal direction vectors are $\mathbf{a}_{r}=\left[1, e^{j 2 \pi d \cos \left(\phi_{r}\right)}, \cdots, e^{j 2 \pi d(N-1) \cos \left(\phi_{r}\right)}\right]^{T}$ and $\mathbf{a}_{t}=$ $\left[1, e^{j 2 \pi d \cos \left(\phi_{t}\right)}, \cdots, e^{j 2 \pi d(M-1) \cos \left(\phi_{t}\right)}\right]^{T}$, where $d$ is the antenna spacing in wavelengths, while $\phi_{r}$ and $\phi_{t}$ refer to the Angle of Arrival (AoA) and Angle of Departure (AoD) with respect to a reference direction, which is defined as geographical North in LTE-Advanced [47]. The $(M \times N)$ scattered elements in $\mathbf{H}_{n}^{S}$ are generated by Clarke's model associated with the power $\sigma_{S}^{2}$. The power normalization requires $\sigma_{D}^{2}+\sigma_{S_{2}}^{2}=1$, and the overall Ricean $K$-factor is defined as $K=\frac{\sigma_{D}^{2}}{\sigma_{S}^{2}}$, where $K=0$ and $K=\infty$ refer to the cases of Rayleigh and AWGN, respectively. Moreover, the channel phase $\theta$ in (2) is uniformly distributed in the interval $(-\pi, \pi)$. This phase rotation $e^{j \theta}$ observed after the received signal's down-conversion from passband to baseband is assumed to be constant during coherent channel estimation and signal detection [49].

\section{B. Terrestrial Uplink and IoT Scenarios}

In the most up-to-date 5G standard releases [50], [51], the single-carrier uplink waveform is generated by Discrete Fourier Transform-Spread-OFDM (DFT-S-OFDM), where the DFT-based transform precoding and zero padding is performed before the OFDM's Inverse Fast Fourier Transform (IFFT). This results in improved oversampling and pulse-shaping characteristics. The single-carrier mode often invokes the constantenvelope Zadoff-Chu sequences, which are constituted by 
optimized phase rotations. The inherent $0 \mathrm{~dB}$ PAPR in the discrete time domain also results in sufficiently low PAPR for the analog signal at the output of pulse-shaping, which is beneficial for the PAs of the UEs [52], [53], since it relaxes the PA's linearity requirements and improves their powerefficiency.

The current $5 \mathrm{G}$ standard release only supports a single transmission layer for the single-carrier mode. Moreover, a variety of unlicensed networks including Bluetooth, Zigbee and IEEE 802.11 ah as well as 5G IoT enablers of Long Range (LoRa) and NarrowBand IoT (NB-IoT) [54], [55] currently all employ a single TA. Therefore, the single-RF SM scheme may constitute an attractive upgrade-candidate for the future communication systems [56], [57]. However, as the cell radius is steadily reduced especially in the $5 \mathrm{G}$ mmWave range of (24.25 52.6 GHz), the LoS components of (2) tend to become more often encountered. Unfortunately, the associated antenna correlation tends to degrade the reception-quality of the IM bits in the SM scheme, which is considered as a major stumbling block of practical SM deployments [10]. We will demonstrate in Sec. II-C and in Sec. V that SM often performs even worse than its single-TA based PSK counterpart in Ricean fading scenarios. Against this background, in this work, we conceive a new family of STSK schemes that are capable of mitigating this problem by maximizing the diversity gain, while the beneficial $0 \mathrm{~dB}$ PAPR signal transmission and ICIfree signal detection capabilities are retained.

\section{Airframe Shadowing}

The three-dimensional maneuvers including pitch, roll and yaw portrayed in Figs. 3(a) and 3(b) were key to the success of modern aviation. However, the detrimental airframe shadowing is induced by the dynamic maneuvers, where the A2G link may be blocked by the fuselage for as long as 74 seconds [58]. It was reported in [58] that using multiple RAs at the GS is unable to mitigate this problem. This is hazardous, since the UAV may have travelled thousands of meters with a blocked control link. The traditional solution is to employ a pair of TAs radiating the same signal, which however would result in self-interference nulls.

In order to mitigate this problem, Alamouti's G2 STBC was invoked in [18]. More explicitly, when the airframeinduced shadowing is encountered, the Ricean K-factors $K_{1}$ and $K_{2}$ of the two TAs are subjected to non-negative lognormal distribution [58]. Moreover, Fig. 3(a) shows that terrain shadowing may also result in $K_{1} \neq K_{2}$ for low-altitude UAVs. It is demonstrated by Fig. 4 that firstly, SM does not perform well in Ricean fading even in the absence of shadowing, because the fading envelopes of the antennas are not sufficiently different for conveying the IM bits. Secondly, the transmit diversity schemes of Alamouti's G2 STBC and CE-ASTSK are shown to be robust against shadowing in Fig. 4, owing to the fact that STBC and CE-ASTSK exploit the fading envelope differences of the antennas both in the spatial and temporal domains. Moreover, as a single-RF scheme, the CE-ASTSK may employ the same RF chain using the same PA as the single-TA scheme, which is particularly advantageous
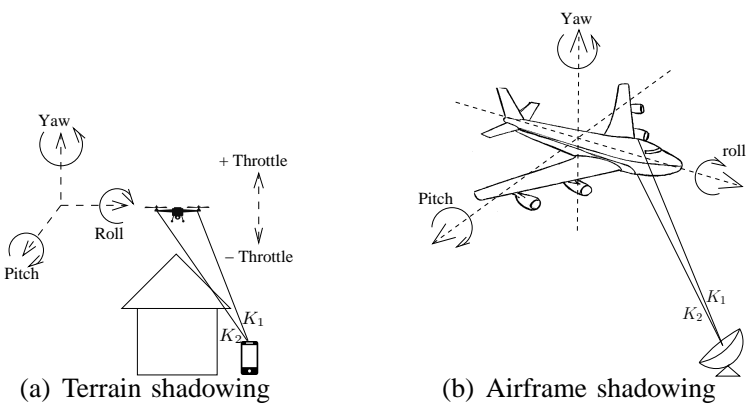

Fig. 3: Terrain shadowing for low-altitude rotary-wing UAV and airframe shadowing for high-altitude fixed-wing UAV.

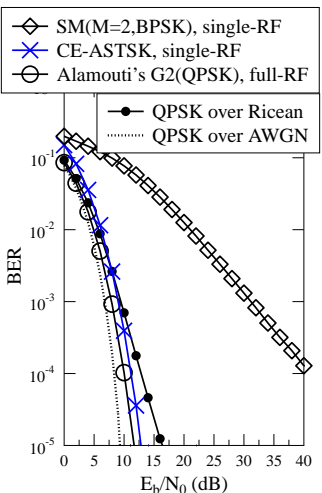

(a) $K_{1}=K_{2}=10 \mathrm{~dB}$

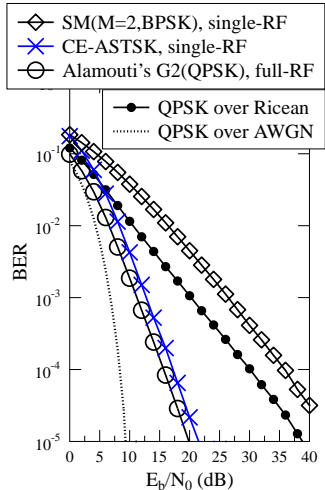

(b) $\left\{K_{1} \neq K_{2}\right\} \in 10-\mid C N(0,10)$
Fig. 4: BER performance comparison between single-/multiple-TA based schemes over Ricean fading and shadowing effect using $M=2$ TAs and $N=1$ RA.

in aerial applications. The details of the CE-ASTSK design will be given in the following sections.

\section{Space-Time Shift Keying Regime}

A variety of high-diversity and high-rate MIMO schemes - including the QO-STBCs [29], [30], the TAST [33], [39] and the division algebra codes, including the Golden code and perfect STBCs [34]-[37] - have been developed for the sake of improving the STBC performance. These MIMO schemes may all be specified in the LDC form [31], [32], [43] of:

$$
\mathbf{S}=\sum_{q=1}^{Q} s_{q} \mathbf{A}_{q}
$$

where a total of $Q$ modulated LPSK/QAM symbols $\left\{s_{q}\right\}_{q=1}^{Q}$ are dispersed both across the spatial and temporal domains by the dispersion matrices $\left\{\mathbf{A}_{q}\right\}_{q=1}^{Q}$. Following the IM philosophy, the LDC's multiplexing form is revised to the STSK's matrix selection form as [20], [21], [43]:

$$
\mathbf{S}=s^{l} \mathbf{A}_{q}
$$

where the classic modulated symbol index $l$ and the dispersion matrix index $q$ carry $\log _{2} L$ and $\log _{2} Q$ bits, respectively. Accordingly, the received signal matrix of (1) is extended as:

$$
\overline{\mathbf{Y}}=\overline{\mathbf{S}} \overline{\mathbf{H}}+\overline{\mathbf{V}}
$$

where we have the vectorized $(1 \times T N)$-element $\overline{\mathbf{Y}}=\operatorname{rvec}(\mathbf{Y})$ and $\overline{\mathbf{V}}=\operatorname{rvec}(\mathbf{V})$. The $(Q \times T N)$-element equivalent channel matrix is given by $\overline{\mathbf{H}}=\bar{\chi}\left(\mathbf{I}_{T} \otimes \mathbf{H}\right)$, where we have the $(Q \times$ $T M$ )-element matrix $\bar{\chi}=\left[\operatorname{rvec}\left(\mathbf{A}_{1}\right)^{T}, \cdots, \operatorname{rvec}\left(\mathbf{A}_{Q}\right)^{T}\right]^{T}$. For the LDCs, the $(1 \times Q)$-element transmitted signal vector 
in (5) is given by $\overline{\mathbf{S}}=\left[s_{1} \cdots s_{Q}\right]$, which is equivalent to $\mathrm{V}$ BLAST associated with $Q$ TAs and $T N$ RAs. Therefore, the LDC receiver has to deal with an increased $\mathrm{ICI}=Q$ streams. By contrast, for the STSK of (4), the $(1 \times Q)$-element vector in (5) is:

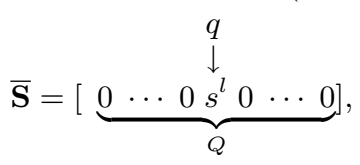

which becomes equivalent to a SM scheme associated with $Q$ TAs and $T N$ RAs, hence the single-stream based ML detectors may be invoked [59], [60].

\section{E. Design Criteria}

The average BER may be expressed as [42], [43], [61]:

$$
\bar{P}_{e, b i t} \leq E\left\{\sum_{i=0}^{I-1} \sum_{\bar{i}=0, \bar{i} \neq i}^{I-1} \frac{d_{H}(i, \bar{i})}{I \log _{2} I} p\left(\mathbf{S}^{i} \rightarrow \mathbf{S}^{\bar{i}}\right)\right\},
$$

where $d_{H}(i, \bar{i})$ refers to the Hamming distance between the bit-mappings of $\mathbf{S}^{i}$ and $\mathbf{S}^{\bar{i}}$, which is directly obtained by conveying the indices $i$ and $\vec{i}$ back to $\log _{2} I$ bits. Furthermore, the Pairwise Error Probability (PEP) in (7) may be expressed as $E\left\{p\left(\mathbf{S}^{i} \rightarrow \mathbf{S}^{\bar{i}}\right)\right\}=E\left\{Q\left[\sqrt{\frac{\left\|\left(\mathbf{S}^{i}-\mathbf{S}^{\bar{i}}\right) \mathbf{H}\right\|^{2}}{2 N_{0}}}\right]\right\}$, where $Q(\cdot)$ represents the integral form of the Q-function. The MIMO schemes operate based on the scattered component $\mathbf{H}_{n}^{S}$ in (2), where the trend of PEP is characterized by the diversity product and by the diversity sum as [44], [62]:

$$
\begin{aligned}
& \Lambda_{p}=\frac{1}{2} \min _{\forall i \neq i^{\prime}} \operatorname{det}(\boldsymbol{\Delta})^{\frac{1}{2 \mathbb{D}(\boldsymbol{\Delta})}} . \\
& \Lambda_{s}=\frac{1}{2 \sqrt{\mathbb{D}(\boldsymbol{\Delta})}} \min _{\forall i \neq i^{\prime}} \operatorname{tr}(\boldsymbol{\Delta})^{\frac{1}{2}} .
\end{aligned}
$$

The distance matrix is defined as $\left[\boldsymbol{\Delta}=\left(\mathbf{S}^{i}-\mathbf{S}^{i^{\prime}}\right)\left(\mathbf{S}^{i}-\mathbf{S}^{i^{\prime}}\right)^{H}\right]$ for $T \leq M$ and $\left[\boldsymbol{\Delta}=\left(\mathbf{S}^{i}-\mathbf{S}^{i^{\prime}}\right)^{H}\left(\mathbf{S}^{i}-\mathbf{S}^{i^{\prime}}\right)\right]$ for $T>M$. For the full-rank $\boldsymbol{\Delta}$, the diversity order in (8) is given by $\mathbb{D}(\boldsymbol{\Delta})=$ $\min (M, T)$. Moreover, according to (7), the average diversity product and average diversity sum are defined as [44]:

$$
\begin{aligned}
& \bar{\Lambda}_{p}=\frac{1}{2}\left[\frac{2}{\log _{2} I+1} \sum_{\forall i} \sum_{\forall i^{\prime} \neq i} \frac{d_{H}\left(i, i^{\prime}\right)}{I \log _{2} I \operatorname{det}(\boldsymbol{\Delta})}\right]^{-\frac{1}{2 \mathbb{D}(\boldsymbol{\Delta})}} . \\
& \bar{\Lambda}_{s}=\frac{1}{2 \sqrt{\mathbb{D}(\boldsymbol{\Delta})}}\left[\frac{2}{\log _{2} I+1} \sum_{\forall i} \sum_{\forall i^{\prime} \neq i} \frac{d_{H}\left(i, i^{\prime}\right)}{I \log _{2} I \operatorname{tr}(\boldsymbol{\Delta})}\right]^{-\frac{1}{2}} .
\end{aligned}
$$

All the four metrics in (8) and (9) are normalized to be within the range of $[0,1]$. Conventionally, the dispersion matrices of LDC and STSK are obtained by maximizing the determinant term of $\Lambda_{p}$. The DSTSK in [44] also invokes $\bar{\Lambda}_{p}$ as the secondary Objective Function (OF). However, it is evidenced by Fig. 5 that the trace terms of $\Lambda_{s}$ and $\bar{\Lambda}_{s}$ become more important for large $N$. Based on this observation, we offer the following improved optimization approach:

Optimization I: In order to achieve the three-fold design objectives of having (I) PAPR $=0 \mathrm{~dB}$ for signal transmission, (II) ICI-free signal detection and (III) maximized diversity gains, our MIMO design follows the STSK model of (4), which aims to satisfy:

$$
\begin{aligned}
& \max _{\left\{\mathbf{A}_{q}\right\}_{q=1}^{Q}} \Lambda_{p} \text { and } \Lambda_{s} \text { and } \bar{\Lambda}_{p} \text { and } \bar{\Lambda}_{s}, \\
& \text { subject to }\left\{\left\{\left|\mathbf{A}_{q}(t, m)\right|^{2}=1\right\}_{t=1}^{T}\right\}_{m=1}^{M} \text { and } \mathbf{A}_{q} \mathbf{A}_{q}^{H}=\mathbf{I}_{T} .
\end{aligned}
$$

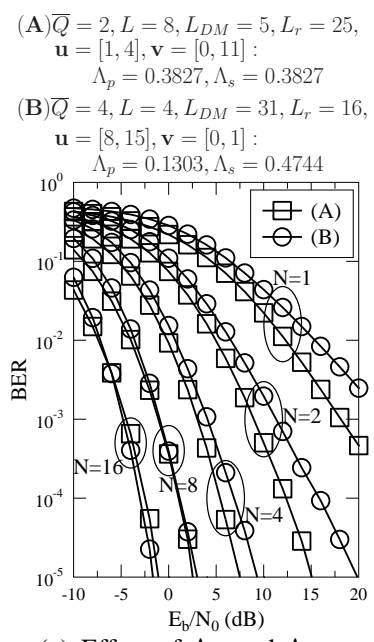

(a) Effect of $\Lambda_{p}$ and $\Lambda_{s}$

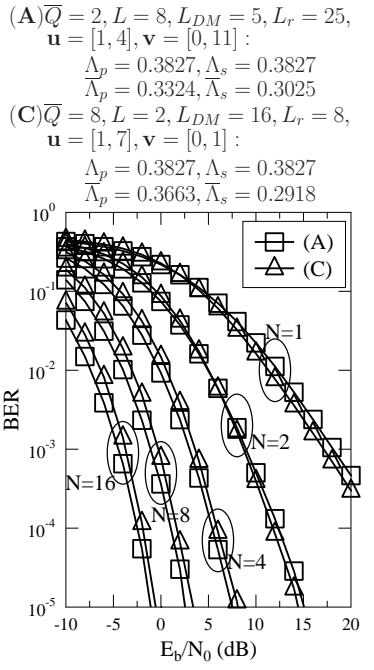

(b) Effect of $\bar{\Lambda}_{p}$ and $\bar{\Lambda}_{s}$
Fig. 5: BER performance comparison between three CE-ASTSK using TAST arrangements associated with $M=T=2$ and $R=2.5$ in Rayleigh fading.

More explicitly, firstly, $\left\{\left\{\left|\mathbf{A}_{q}(t, m)\right|^{2}=1\right\}_{t=1}^{T}\right\}_{m=1}^{M}$ indicates that all elements in the dispersion matrix $\mathbf{A}_{q}$ are taken from the CE PSK constellation. Secondly, $\mathbf{A}_{q} \mathbf{A}_{q}^{H}=\mathbf{I}_{T}$ implies that $\mathbf{A}_{q}$ is unitary, which ensures ICI-free STSK detection [20], [21], [43], [59]. Finally, the four CFs of (10) are not concave in the variable $\mathbf{A}_{q}$, hence typically the gradient-ascent methods are invoked by the conventional LDC and STSK schemes, which often lead to local maxima [20], [21], [31], [32], [43]. However, we will demonstrate in Sec. III that by confining the elements of $\mathbf{A}_{q}$ to be taken from a finite-cardinality $L_{D M^{-}}$ PSK constellation, the brute-force exhaustive search for the global maxima becomes feasible, as specified by Remark 1 of Sec. III-A.

Moreover, the four OFs in (10) cannot always be all maximized at the same time, as exemplified by Fig. 5. Therefore, we propose that for small $N$, the maximization of $\Lambda_{p}$ is given the highest priority, followed by $\Lambda_{s}, \bar{\Lambda}_{p}$ and $\bar{\Lambda}_{s}$. By contrast, the highest priority is given to $\Lambda_{s}$ for large $N$, followed by $\Lambda_{p}, \bar{\Lambda}_{s}$ and $\bar{\Lambda}_{p}$. For example, when considering candidates (A) and (B) of Fig. 5(a), candidate (A) and candidate (B) associated with a higher $\Lambda_{p}$ and $\Lambda_{s}$ are chosen for small and large $N$, respectively. Moreover, for the situation of two candidates having the same $\Lambda_{p}$ and $\Lambda_{s}$ as seen in Fig. 5(b), candidate (C) and candidate (A) associated with higher $\bar{\Lambda}_{p}$ and $\bar{\Lambda}_{s}$ are chosen for small and large $N$, respectively.

\section{Single-RF Constant-EnVElope Design}

In this section, we propose the single-RF CE-ASTSK, where the TAST, PAST and DR arrangements are devised in Secs. III-A, III-B and III-C, respectively.

\section{A. Threaded Algebraic Space-Time (TAST) Scheme}

For the proposed CE-ASTSK, the dispersion elements in $\mathbf{A}_{q}$ of (4) operate based on the $L_{D M}$-th root of unity $\left[\omega_{L_{D M}}=\right.$ $\left.\exp \left(j \frac{2 \pi}{L_{D M}}\right)\right]$, which are taken from the following $(T \times \bar{Q})$ element Vandermonde generator matrix: 


$$
\begin{aligned}
\mathbf{G}_{A} & =\operatorname{VDM}_{T \times \bar{Q}}\left(\omega_{L_{D M}}^{u_{1}}, \cdots, \omega_{L_{D M}}^{u_{T}}\right) \\
= & {\left[\begin{array}{ccccccc}
1 & \omega_{L_{D M}}^{u_{1}} & \omega_{L_{D M}}^{2 u_{1}} & \ldots & \omega_{L_{D M}}^{(\bar{q}-1) u_{1}} & \ldots & \omega_{L_{D M}}^{(\bar{Q}-1) u_{1}} \\
1 & \omega_{L_{D M}}^{u_{2}} & \omega_{L_{D M}}^{2 u_{2}} & \ldots & \omega_{L_{D M}}^{(\bar{q}-1) u_{2}} & \ldots & \omega_{L_{D M}}^{(\bar{Q}-1) u_{2}} \\
\vdots & \vdots & \vdots & & \vdots & & \vdots \\
1 & \omega_{L_{D M}}^{u_{T}} & \omega_{L_{D M}}^{2 u_{T}} & \ldots & \omega_{L_{D M}}^{(\bar{q}-1) u_{T}} & \ldots & \omega_{L_{D M}}^{(\bar{Q}-1) u_{T}}
\end{array}\right] . }
\end{aligned}
$$

More explicitly, as an intermediate step, the $(T \times M)$-element CE-ASTSK using the Diagonal Algebraic Space-Time (DAST) scheme associated with $M=T$ is constructed as:

$$
\mathbf{S}=s^{l} \overline{\mathbf{A}}_{\bar{q}}=s^{l} \operatorname{diag}\left(\left[\omega_{L_{D M}}^{(\bar{q}-1) u_{1}}, \omega_{L_{D M}}^{(\bar{q}-1) u_{2}}, \cdots, \omega_{L_{D M}}^{(\bar{q}-1) u_{T}}\right]\right),
$$

where the diagonal matrix $\overline{\mathbf{A}}_{\bar{q}}$ is formed by taking the $\bar{q}$-th column in $\mathbf{G}_{A}$ of (11). Furthermore, instead of only using the diagonal matrix, the CE-ASTSK using TAST is constructed as:

$$
\mathbf{S}=s^{l} \mathbf{A}_{\tau, \bar{q}}=s^{l} \phi_{\tau} \overline{\mathbf{A}}_{\bar{q}} \mathbf{G}_{r}^{\tau-1}
$$

where the dispersion matrix is now given by $\left\{\left\{\mathbf{A}_{\tau, \bar{q}}=\right.\right.$ $\left.\left.\phi_{\tau} \operatorname{diag}\left(\left[\omega_{L_{D M}}^{(\bar{q}-1) u_{1}}, \cdots, \omega_{L_{D M}}^{(\bar{q}-1) u_{T}}\right]\right) \mathbf{G}_{r}^{\tau-1}\right\}_{\bar{q}=1}^{\bar{Q}}\right\}_{\tau=1}^{T}$. The TAST of (13) partitions a $(T \times T)$-element space-time matrix into $T$ non-overlapping threads, of which only a sinlge thread is activated. The associated $(T \times T)$-element thread-switching matrix in (13) is given by:

$$
\mathbf{G}_{r}=\left[\begin{array}{cccc}
0 & \cdots & 0 & 1 \\
1 & \cdots & 0 & 0 \\
\vdots & \ddots & \vdots & \vdots \\
0 & \cdots & 1 & 0
\end{array}\right]
$$

An example of (14) for $T=4$ is presented in Table II. In summary, the modulation index $l$, the dispersion matrix index $\bar{q}$ and the thread-switching index $\tau$ in (13) convey $\log _{2} L$, $\log _{2} \bar{Q}$ and $\log _{2} T$ bits, respectively. The overall throughput is given by $R=\frac{\log _{2} L+\log _{2} \bar{Q}+\log _{2} T}{T}$.

In contrast to the DSTSK of [44], first of all, the coherent CE-ASTSK no longer requires $L_{D M}$ and $L_{r}$ to be a power of two. Secondly, we revise the phase rotations in (13) as $\left\{\phi_{\tau}=\omega_{L_{r}}^{v_{\tau}}\right\}_{\tau=1}^{T}$, where a new vector of integer parameters $\mathbf{v}=\left[v_{1}, \cdots, v_{T}\right]$ is introduced. As for the degree of freedom, it is sensible to choose $\left(\bar{Q} \leq L_{D M} \leq L \bar{Q} T\right)$ and $\left(T \leq L_{r} \leq\right.$ $L \bar{Q} T)$. In summary, the design guidelines for (13) are:

(I) When LPSK is employed for $s^{l}=\omega_{L}^{l}$, the transmitted signals in (13) are always in the form of $\omega_{L}^{l} \omega_{L_{D M}}^{(\bar{q}-1) u_{t}} \omega_{L_{r}}^{v_{\tau}}$, which results in PAPR $=0 \mathrm{~dB}$ for signal transmission.

(II) Since (13) follows the STSK form of (4), the CEASTSK using TAST is also ICI-free. The total number of dispersion matrices in (4) is now given by $Q=\bar{Q} T$

(III) The optimization of (10) becomes equivalent to searching for the best integers $\left\{u_{t} \in\left[1, L_{D M}\right)\right\}_{t=1}^{T}$ and $\left\{v_{\tau} \in\left[0, L_{r}\right)\right\}_{\tau=1}^{T}$ that maximize the diversity gains in (10).

Since Optimization I requires the maximization of all four OFs in (10), the simplified evaluation of $\Lambda_{p}$ in [44] does not reduce the search space. Nonetheless, Propositions 1-3 of [44] still remain valid, where the term $\frac{T \pi(\tau-1)}{L_{r}}$ in (26) of [44] is now replaced by $\frac{T \pi v_{\tau}}{L_{r}}$. Consequently, we require $\left\{u_{t}\right\}_{t=1}^{T}$ and $\left\{v_{\tau}\right\}_{\tau=1}^{T}$ to be relatively prime to $L_{D M}$ and $L_{r}$, respectively, so that $\Lambda_{p}=0$ is avoided. Moreover, we always set $v_{1}=0$ so that $\phi_{1}=1$. This is because the parameters $\left\{v_{\tau}\right\}_{\tau=1}^{T}$ are only responsible for maximizing the phase differences between $\left\{\phi_{\tau}\right\}_{\tau=1}^{T}$, where the value of $\phi_{1}$ does not have any impact.

Remark 1 (Parameters): The CE-ASTSK using the TAST scheme of (13) is unambiguously specified by the integer parameters of $\left(M, N, T, \bar{Q}, L, L_{D M}, \mathbf{u}, L_{r}, \mathbf{v}\right)$. As a result, the CE-ASTSK has the following advantages over the conventional LDC and STSK. First of all, the brute-force exhaustive search over all eligible integers becomes feasible for (10). The search spaces for $\mathbf{u}=\left[u_{1}, \cdots, u_{T}\right]$ and $\mathbf{v}=\left[v_{1}, \cdots, v_{T}\right]$ are bounded by $\left(\frac{L_{D M}}{2}\right)^{T}$ and $\left(\frac{L_{r}}{2}\right)^{T-1}$, respectively. Secondly, the CE-ASTSK transceivers only have to store the integer parameters instead of the full signal matrices

Remark 2 (Complexity): As a ICI-free STSK scheme, the signal detection complexity of CE-ASTSK using the TAST scheme of (13) does not grow with modulation alphabet size $L$ [59], [60]. Hence, for a specific throughput requirement of $R=\frac{\log _{2} L \bar{Q} T}{T}$, it is favourable to choose a small $\bar{Q}$ and a large $L$ over all combinations of $\bar{Q} L=\frac{2^{R T}}{T}$.

Example 1: For the case of $M=T=2$ at a throuhgput of $R=2.0$, we have performed a comprehensive fullsearch over all possible combinations of parameters that satisfy $L \bar{Q}=8, \bar{Q} \leq L_{D M} \leq L \bar{Q} T$ and $T \leq L_{r} \leq L \bar{Q} T$. The best solution found by Optimization I has the parameters of $\left(\bar{Q}, L, L_{D M}, \mathbf{u}, L_{r}, \mathbf{v}\right)=(4,2,8,[1,3], 8,[0,1])$. According to the paramters, the signal matrix of (13) is contructed as $\mathbf{S}=\omega_{2}^{l} \omega_{8}^{\tau-1}\left[\begin{array}{ll}\omega_{8} & 0 \\ 0 & \omega_{8}^{3}\end{array}\right]^{\bar{q}-1}\left[\begin{array}{ll}0 & 1 \\ 1 & 0\end{array}\right]^{\tau-1}$ associated with $l \in$ $\{0,1\}, \bar{q} \in\{1,2,3,4\}$ and $\tau \in\{1,2\}$. The resultant diversity gains of (8) are given by $\left(\Lambda_{p}=0.5946, \Lambda_{s}=0.7071\right)$, which are substantially higher than $\left(\Lambda_{p}=\Lambda_{s}=0.5\right)$ of Alamouti's G2 STBC using QPSK [25]. Our detailed performance comparisons will be presented in Sec V.

Example 2: For using $M=T=4$ at $R=$ 1.0, Optimization I produces the TAST parameters of $\left(\bar{Q}, L, L_{D M}, \mathbf{u}, L_{r}, \mathbf{v}\right)=(2,2,4,[1,1,1,1], 16,[0,1,2,3])$ that achieve $\left(\Lambda_{p}=0.5453, \Lambda_{s}=0.7071\right)$. These diversity gains are higher than $\left(\Lambda_{p}=\Lambda_{s}=0.5\right)$ of both the 1/2-rate STBC using QPSK and of QO-STBC using BPSK, where the former relies on $T=8$ [26], while the latter imposes the ICI of 2 streams [29], [30].

According to Remark 2, the search space of Optimization I grows exponentially both with $R$ and $T$. In order to simplify this, we further conceive a hierarchical approach as follows:

Optimization II: When the full-search for the CE-ASTSK relying on the TAST philosophy becomes infeasible at a high throughput $R$, we firstly invoke (10) of Optimization I for the DAST of (12) associated with $\bar{Q} L=\frac{2^{R T}}{T}$, which produces the parameters of $L_{D M}$ and $\mathbf{u}$. Following this, (10) of Optimization I is invoked again for the TAST of (13) in order to further obtain $L_{r}$ and $\mathbf{v}$. The detailed step-by-step description is formulated in Algorithm 1.

Example 3: For $M=T=2$ and $R=3.0$, we have $\bar{Q} L=$ 32. Let us firstly invoke (10) of Optimization I for the DAST of (12). For small $N$, we obtain candidate (A) associated with the parameters of $\left(\bar{Q}, L, L_{D M}, \mathbf{u}\right)=(4,8,11,[1,10])$ that achieves $\left(\Lambda_{p}=0.259, \Lambda_{s}=0.2817\right)$. For large $N$, we obtain 


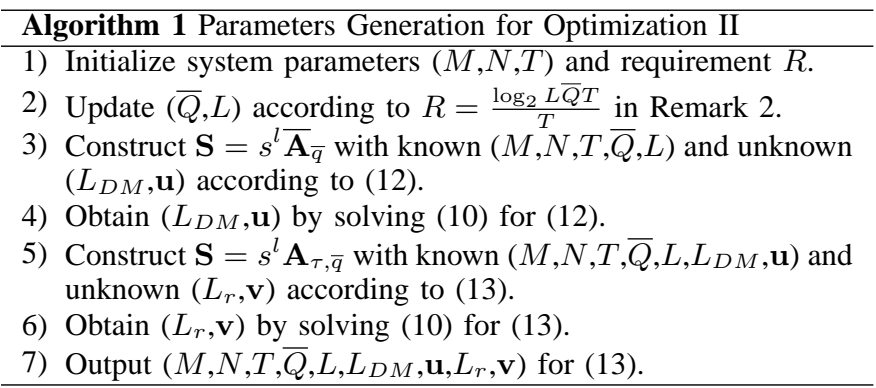

the candidate (B) of $\left(\bar{Q}, L, L_{D M}, \mathbf{u}\right)=(4,8,32,[3,27])$ that achieves $\left(\Lambda_{p}=0.2494, \Lambda_{s}=0.3827\right)$. In other words, candidates (A) and (B) are chosen for their highest $\Lambda_{p}$ and $\Lambda_{s}$, respectively. Following this, (10) of Optimization I is invoked again for the TAST of (13) in order to further obtain $L_{r}$ and v. Accordingly, for small $N$, the parameters of candidate (A) are complemented by $\left(L_{r}, \mathbf{v}\right)=(16,[0,1])$, which results in $\left(\Lambda_{p}=0.259, \Lambda_{s}=0.2817\right)$ for (13). Similarly, for large $N$, candidate (B) is complemented by $\left(L_{r}, \mathbf{v}\right)=(64,[0,1])$, which results in $\left(\Lambda_{p}=0.2214, \Lambda_{s}=0.3827\right)$ for (13). Comparing to $\left(\Lambda_{p}=\Lambda_{s}=0.2706\right)$ of Alamouti's G2 STBC using 8PSK [25], candidate (B) achieves $\Lambda_{s}=0.3827$, which indicates a better performance, when a large $N$ is used.

\section{B. Permuted Algebraic Space-Time (PAST) Scheme}

In order to maximize the IM spectral efficiency, we propose the PAST design, which allows full permutation of all signal positions. The $(T \times M)$-element signal matrix is contructed for $M=T$ as:

$$
\mathbf{S}=s^{l} \mathbf{A}_{p, \bar{q}}=s^{l} \phi_{p} \overline{\mathbf{A}}_{\bar{q}} \overline{\mathbf{G}}_{p},
$$

where the dispersion matrix is $\left\{\left\{\mathbf{A}_{p, \bar{q}}=\right.\right.$ $\left.\left.\phi_{p} \operatorname{diag}\left(\left[\omega_{L_{D M}}^{(\bar{q}-1) u_{1}}, \cdots, \omega_{L_{D M}}^{(\bar{q}-1) u_{T}}\right]\right) \overline{\mathbf{G}}_{p}\right\}_{\bar{q}=1}^{\bar{Q}}\right\}_{p=1}^{\mathcal{P}}$, while we have $\left\{\phi_{p}=\omega_{L_{r}}^{v_{p}}\right\}_{p=1}^{\mathcal{P}}$. For a $(T \times T)$-element sparse signal space, there are a total of $\mathcal{P}=2^{\left(\left\lfloor\log _{2} T !\right\rfloor\right)}$ legitimate signal positions, which carries $\left\lfloor\log _{2} T !\right\rfloor$ IM bits. The index $p$ in (15) specifies a $T$-element index vector $\mathbf{a}_{p}$, which contains the permutated positions of $\{1, \cdots, T\}$. Therefore, the element on the $r$-th row and $c$-th column in $\overline{\mathbf{G}}_{p}$ is:

$$
\overline{\mathbf{G}}_{p}(r, c)=\left\{\begin{array}{ll}
1, & \text { if } \mathbf{a}_{p, r}=c \\
0, & \text { otherwise }
\end{array} .\right.
$$

The difference between the definition of TAST in (14) and PAST in (16) is exemplified in Table II for $T=4$, where the TAST's thread-switching matrix and the PAST's permutation matrix convey $\log _{2} T=2$ and $\left\lfloor\log _{2} T !\right\rfloor=4$ bits, respectively.

In summary, for the CE-ASTSK using the PAST of (15), the modulation index $l$, the dispersion matrix $\bar{q}$ and the permutation index $p$ convey $\log _{2} L, \log _{2} \bar{Q}$ and $\left\lfloor\log _{2} T !\right\rfloor$ bits, respectively. Therefore, the throughput is improved to $R=\frac{\log _{2} L+\log _{2} \bar{Q}+\left\lfloor\log _{2} T !\right\rfloor}{T}$. Similar to TAST, Optimization I and Optimization II may be invoked for (15) at low and high throughputs, respectively.

Example 4: For the case of $M=T=4$ at throughput $R=2.0$, we invoke Optimization II for (15) and obtain three candidates associated with different combinations of $\bar{Q}$ and $L$. The parameters
TABLE II: Comparison between the TAST's thread-switching matrix $\left\{\overline{\mathbf{G}}_{r}^{\tau-1}\right\}_{\tau=1}^{T}$ of (14) and the PAST's permutation matrix $\left\{\overline{\mathbf{G}}_{p}\right\}_{p=1}^{\mathcal{P}}$ of (16) for $T=4$.

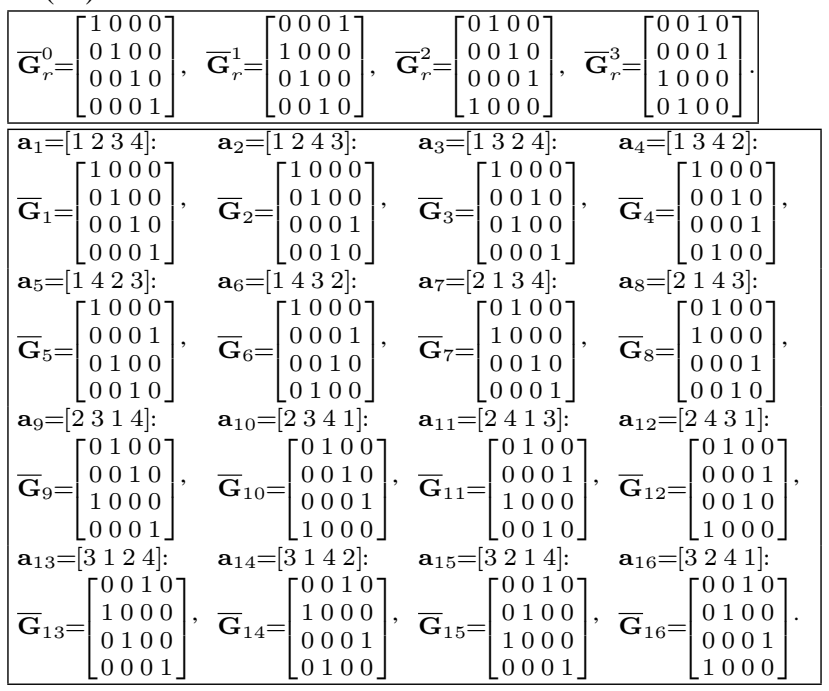

of candidate (A) are given by $\left(\bar{Q}, L, L_{D M}, \mathbf{u}, L_{r}, \mathbf{v}\right)=$ $(2,8,43,[3,14,29,40], 256,[0,36,53,202,60,182,39,82,126,101$, $56,76,145,2,183,235])$ and the diversity gains are $\left(\Lambda_{p}=0.1031, \Lambda_{s}=0.3827\right)$. The corresponding parameters for candidate (B) are $(4,4,16,[1,5,9,13], 2048,[0,382,279,16,332$, $1445,800,1176,341,82,521,1729,1928,1752,194,932])$, which achieve $\left(\Lambda_{p}=0.1093, \Lambda_{s}=0.5017\right)$. For candidate (C), the parameters are $(8,2,16,[1,3,5,7], 2048,[0,1805,955,1571,351,1112,1681,1213$, $1185,894,584,461,84,1942,846,566])$, which achieve $\left(\Lambda_{p}=0.1458, \Lambda_{s}=0.5043\right)$. According to Remark 2, candidate (A) exhibits the lowest STSK detection complexity, followed by candidates (B) and (C). By contrast, candidate (C) achieves the highest diversity gains, followed by candidates (B) and (A). At the same $R$, the $1 / 2$-rate STBC using $T=8$ [26] achieves $\left(\Lambda_{p}=\Lambda_{s}=0.138\right)$ and $\left(\Lambda_{p}=\Lambda_{s}=0.2236\right)$ for employing 16PSK and square 16QAM, respectively, where the latter imposes a higher PAPR $=2.55 \mathrm{~dB}$. Moreover, the QOSTBC using QPSK [29], [30] achieves $\left(\Lambda_{p}=\Lambda_{s}=0.3536\right)$ at the cost of ICI $=2$. In summary, all the three CE-ASTSK candidates achieve unequivocally higher $\Lambda_{s}$ than the STBCs, which once again indicates a better performance for large $N$.

\section{Diversity-Rate (DR) Tradeoff}

In order to use $T<M$ for large $M$, first of all, we propose the Type I DR arrangement. The $(T \times M)$-element signal matrix is partitioned into $W=M / T$ number of nonoverlapping $(T \times T)$-element submatrices, where a single one out of $W$ is activated:

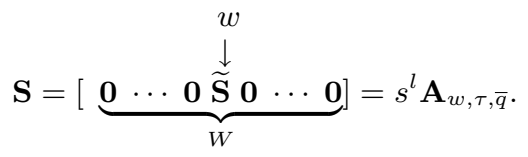

The $(T \times T)$-element submatrix $\widetilde{\mathbf{S}}$ in (17) may invoke the TAST scheme of (13). Accordingly, the dispersion matrix in (17) is $\mathbf{A}_{w, \tau, \bar{q}}=\left[\begin{array}{llllll}\mathbf{0} & \cdots & \mathbf{0} & \widetilde{\mathbf{A}}_{\tau, \bar{q}} \mathbf{0} & \cdots & \mathbf{0}\end{array}\right]$, where $\widetilde{\mathbf{A}}_{\tau, \bar{q}}$ refers to the TAST dispersion matrix defined in (13). The resultant throughput of (17) is $R=\frac{\log _{2} L+\log _{2} \bar{Q}+\log _{2} T+\log _{2} W}{T}$, where an extra number of $\log _{2} W$ IM bits are conveyed. 
The Type I DR of (17) does not require any further optimization, yet the three-fold benefits of single-RF, PAPR = $0 \mathrm{~dB}$ and ICI-free features are all retained. We note that (17) can also invoke the PAST of (15) as the component submatrix. Since TAST and PAST only differ for $T>2$, we focus our attention on the $T=2$ scenario.

Example 5: For the case of using $M=4$ and $T=2$ at $R=3.0$, the Type I CE-ASTSK-DR of (17) invokes the TAST of (13) optimized for $M=$ $T=2$ and $\bar{Q} L=16$, which has two candidates that achieve the highest $\Lambda_{p}$ and $\Lambda_{s}$, respectively. The candidate (A) has the parameters of $\left(\bar{Q}, L, L_{D M}, \mathbf{u}, L_{r}, \mathbf{v}\right)=$ $(2,8,21,[4,17], 25,[0,11])$, and the candidate (B) has $\left(\bar{Q}, L, L_{D M}, \mathbf{u}, L_{r}, \mathbf{v}\right)=(8,2,18,[1,5], 12,[0,1])$. Accordingly, the $(2 \times 2)$-element submatrix in (17) is constructed based on (13) as $\widetilde{\mathbf{S}}=s^{l} \omega_{L_{r}}^{v_{\tau}} \operatorname{diag}\left(\left[\omega_{L_{D M}}^{(\bar{q}-1) u_{1}}, \omega_{L_{D M}}^{(\bar{q}-1) u_{2}}\right]\right) \mathbf{G}_{r}^{\tau-1}$. The resultant CE-ASTSK-DR of (17) forms $(2 \times 4)$-element codewords of $\left\{\left[\begin{array}{ll}\mathbf{0} & \widetilde{\mathbf{S}}\end{array}\right],\left[\begin{array}{ll}\widetilde{\mathbf{S}} & \mathbf{0}\end{array}\right]\right\}$, which achieve $\left(\Lambda_{p}=\Lambda_{s}=\right.$ $0.3827)$ and $\left(\Lambda_{p}, \Lambda_{s}\right)=(0.3647,0.5)$ for candidates (A) and (B), respectively. By contrast, the 3/4-rate STBC [26]-[28] achieves $\left(\Lambda_{p}=\Lambda_{s}=0.1126\right)$ and $\left(\Lambda_{p}=\Lambda_{s}=0.1826\right)$ for using 16PSK and square 16QAM, respectively. Moreover, the QO-STBC using 8PSK [29], [30] has $\left(\Lambda_{p}, \Lambda_{s}\right)=$ $(0.1674,0.1913)$. Therefore, both DR candidates (A) and (B) achieve substantially higher diversity gains than the STBCs without either increasing PAPR or imposing ICI.

In order to improve the throughput, we proceed to conceive the Type II design, which allows all the legitimate $\left.\left.\mathcal{C}=2^{\left\lfloor\log _{2}\left(T^{\prime} \rho\right.\right.}\right)\right\rfloor$ combinations of signal positions seen in [6]. Specifically, the signal matrices for $M=4$ and $T=2$ are:

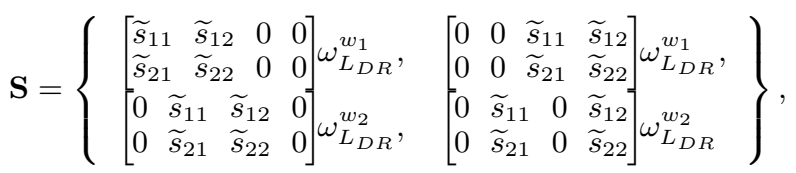

where $\left\{\left\{\widetilde{s}_{r, c}\right\}_{r=1}^{T}\right\}_{c=1}^{T}$ are signals in the $(T \times T)$-element TAST matrix $\widetilde{\mathbf{S}}$ of (13), while the codewords associated with overlapping signal positions are assigned new phase rotations $\left\{\omega_{L_{D R}}^{w_{c}}\right\}_{c=1}^{\mathcal{C} / T}$. In summary, the Type II DR throughput is $R=$ $\frac{\log _{2} L+\log _{2} \bar{Q}+\log _{2} T+\log _{2} \mathcal{C}}{T}$. For the case of $M=4$ and $T=2$, Type II maps $\log _{2} \mathcal{C}=2$ bits to the signal positions, which is higher than $\log _{2} W=1$ of (17). The price paid is that further search across the set of $\left\{\omega_{L_{D R}}^{w_{c}}\right\}_{c=1}^{\mathcal{C} / T}$ is required. Similarly to Optimization II for TAST, we set $w_{1}=0$ and $L_{D R} \leq \bar{Q} L \mathcal{C}$. The following three-step hierarchical optimization approach is conceived for the Type II DR as:

Optimization III: For a specific $R$, the first step is to invoke (10) of Optimization I for the DAST of (12) associated with $\bar{Q} L=\frac{2^{R T}}{T C}$, which produces the parameters of $L_{D M}$ and $\mathbf{u}$. Secondly, (10) is invoked again for the TAST of (13) in order to further obtain $L_{r}$ and v. Finally, the Type II DR once again invokes (10) for $L_{D R}$ and $\mathbf{w}$.

Example 6: For the case of $M=4$ and $T=2$ at $R=4.0$, Optimization III produces two legitimate candidates associated with $\left(\bar{Q}, L, L_{D M}, \mathbf{u}, L_{r}, \mathbf{v}, L_{D R}, \mathbf{w}\right)=$ $(4,8,32,[1,25], 64,[0,1], 128,[0,1])$ $(16,2,32,[1,7], 16,[0,1], 64,[0,1])$, $\left(\Lambda_{p}, \Lambda_{s}\right)=(0.1317,0.3827)$ which achieve and $\left(\Lambda_{p}, \Lambda_{s}\right) \quad=$
TABLE III: Signal matrices of single-RF CE-ASTSK and fullRF CE-STSK associated with parameters $M=T=2$ and $\left(\bar{Q}, L, L_{D M}, \mathbf{u}, L_{r}, \mathbf{v}\right)=(2,2,4,[1,3], 4,[0,1])$ at $R=1.5$.

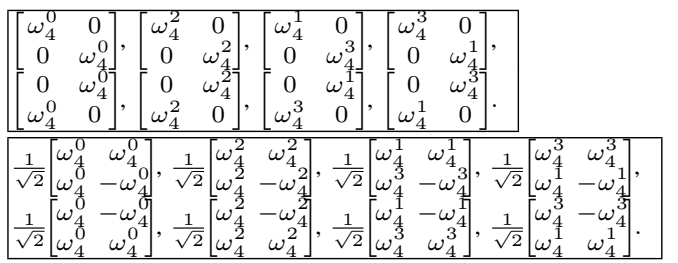

$(0.1863,0.3827)$, respectively. By contrast, the QOSTBC [29], [30] has the much lower values of $\left(\Lambda_{p}, \Lambda_{s}\right)=(0.0609,0.0975)$ and $\left(\Lambda_{p}=\Lambda_{s}=0.1581\right)$ for using 16PSK and 16QAM, respectively.

\section{Full-RF And Reduced-RF Constant-EnVElope DESIGN}

In this section, we propose the full-RF and reduced-RF CESTSK in Secs. IV-A and IV-B, respectively, while the reducedRF CE-GSTSK is conceived in Sec. IV-C.

\section{A. Full-RF Space-Time Shift Keying}

It has been demonstrated by Examples 1-6 that the singleRF CE-ASTSK of Sec. III is capable of outperforming the full-RF orthognal STBCs [25]-[28] without either increasing the PAPR or imposing ICI. Therefore, it is beneficial to employ CE-STSK in the same full-RF setup as the STBCs using the same PAs. Against this background, we propose the following lossless transformation between single-RF CE-ASTSK and full-RF CE-STSK:

Proposition I: The full-RF CE-STSK is obtained upon multiplying the $(T \times M)$-element CE-ASTSK signal matrix by a $(M \times M)$-element Hadamard matrix normalized by $\frac{1}{\sqrt{M}}$, where the triple benefits of PAPR $=0 \mathrm{~dB}$, ICI-free reception and unaffected diversity gains are retained.

Proof. The well-known Hadamard matrix is an orthogonal matrix constituted by elements of \pm 1 . The CE-ASTSK signal matrix is sparse, having a single PSK signal on each row and column. Therefore, the multiplication of the two matrices does not induce any signal additions, and the resultant signals are still drawn from the same PSK constellation associated with PAPR $=0 \mathrm{~dB}$. From the receiver's point of view, the orthogonal Hadamard matrix does not change the characteristics of the fading matrix $\mathbf{H}_{n}$ of (1). Therefore, the detection is still performed on the sparse data-carrying matrix, which remains ICI-free and retains undiminished diversity gains.

For example, the Hadamard matrix combined with power normalization for $M=2$ is given by $\frac{1}{\sqrt{2}}\left[\begin{array}{cc}1 & 1 \\ 1 & -1\end{array}\right]$. The CE-ASTSK using TAST of (13) is in the form of $\left\{\left[\begin{array}{ll}s_{1} & 0 \\ 0 & s_{2}\end{array}\right],\left[\begin{array}{cc}0 & s_{1} \\ s_{2} & 0\end{array}\right]\right\}$, where both $s_{1}$ and $s_{2}$ are taken from the PSK constellation. Upon applying Proposition I, the resultant CE-TAST is in the form of $\left\{\frac{1}{\sqrt{2}}\left[\begin{array}{cc}s_{1} & s_{1} \\ s_{2} & -s_{2}\end{array}\right], \frac{1}{\sqrt{2}}\left[\begin{array}{cc}s_{1} & -s_{1} \\ s_{2} & s_{2}\end{array}\right]\right\}$, which retains PAPR = $0 \mathrm{~dB}$. Table III further exemplifies the signal matrices of CEASTSK and CE-STSK associated with the parameters $M=$ 
$T=2$ and $\left(\bar{Q}, L, L_{D M}, \mathbf{u}, L_{r}, \mathbf{v}\right)=(2,2,4,[1,3], 4,[0,1])$ at $R=1.5$.

Moreover, for $M=2$, the STSK receiver may treat $\frac{1}{\sqrt{2}}\left[\begin{array}{cc}1 & 1 \\ 1 & -1\end{array}\right]\left[\begin{array}{l}\mathbf{H}_{n}^{1} \\ \mathbf{H}_{n}^{2}\end{array}\right]=\frac{1}{\sqrt{2}}\left[\begin{array}{l}\mathbf{H}_{n}^{1}+\mathbf{H}_{n}^{2} \\ \mathbf{H}_{n}^{1}-\mathbf{H}_{n}^{2}\end{array}\right]$ as the equivalent fading matrix, where $\mathbf{H}_{n}^{1}$ and $\mathbf{H}_{n}^{2}$ refers to the 1st and 2nd row in $\mathbf{H}_{n}$ of (1). For the case of Rayleigh fading, both rowvectors $\mathbf{H}_{n}^{1}+\mathbf{H}_{n}^{2}$ and $\mathbf{H}_{n}^{1}-\mathbf{H}_{n}^{2}$ are still zero-mean Gaussiandistributed. However, for a Ricean fading having a mean of $\sigma_{D}$ in (2), the two row-vectors are still Gaussian-distributed but their means become $2 \sigma_{D}$ and 0 , respectively. Nonetheless, the average mean of the whole matrix $\mathbf{H}_{n}$ remains $\sigma_{D}$. We will demonstrate in Secs. V and VI that Proposition I is valid for both Rayleigh and Ricean fading channels.

\section{B. Reduced-RF Space-Time Shift Keying}

Following Proposition I, we propose to transform the singleRF CE-ASTSK-DR of Sec. III-C into the reduced-RF CESTSK-RH. More explicity, we offer the following corollary:

Corollary I: When a reduced number of $M_{A}=T$ out of $M$ TAs are activated, the $(T \times M)$-element CE-STSK-RH is obtained by replacing the $(T \times T)$-element sparse CE-ASTSK submatrix in the DR arrangements of Sec. III-C by its fullRF $(T \times T)$-element CE-STSK submatrix, which invokes a $\left(M_{A} \times M_{A}\right)$-element Reduced-size Hadamard (RH) matrix. Consequently, the CE-STSK-RH retains the triple benefits of PAPR $=0 \mathrm{~dB}$, ICI-free reception and undiminished diversity gains as the CE-ASTSK-DR of Sec. III-C.

Example 7: For the case of $M=4$ and $T=2$ at $R=3.0$, a reduced $M_{A}=T=2$ number of RF chains are activated. Optimization III in Sec. III-C produces the parameters $\left(\bar{Q}, L, L_{D M}, \mathbf{u}, L_{r}, \mathbf{v}, L_{D R}, \mathbf{w}\right)=$ $(4,2,8,[1,3], 8,[0,1], 16,[0,1])$ for the Type II of CEASTSK-DR. Following this, the $(2 \times 4)$-element reduced-RF CE-STSK-RH is still expressed by (18), but the $(2 \times 2)$ element submatrix in (18) is now given by the TAST of (13) multiplified by the normalized RH matrix $\left[\begin{array}{cc}1 & 1 \\ 1 & -1\end{array}\right]$ as $\widetilde{\mathbf{S}}=$ $\frac{s^{l} \omega_{8}^{\tau-1}}{\sqrt{2}}\left[\begin{array}{cc}\omega_{8} & 0 \\ 0 & \omega_{8}^{3}\end{array}\right]^{\bar{q}-1}\left[\begin{array}{ll}0 & 1 \\ 1 & 0\end{array}\right]^{\tau-1}\left[\begin{array}{cc}1 & 1 \\ 1 & -1\end{array}\right]$. The reduced-RF CE-STSKRH achieves $\left(\Lambda_{p}, \Lambda_{s}\right)=(0.3417,0.5187)$. By contrast, the STBC-SM using QPSK [6] achieves $\left(\Lambda_{p}, \Lambda_{s}\right)=(0.4598,0.5)$. Therefore, the proposed CE-STSK-RH performs better for large $N$.

\section{Reduced-RF Generalized Space-Time Shift Keying}

Compared to Examples 1-6, the performance advantage of the reduced-RF STSK in Example 7 becomes less substantial. This is because the STBC-SM [6], [38] has already improved the spectral efficiency of Alamouti's G2 STBC by the IM design, which leaves little room for further improvement either without increasing $T$ [63] or without imposing ICI [64]-[66]. Against this background, we propose the reduced-RF CEGSTSK, which retains both the $0 \mathrm{~dB}$ PAPR and the minimal delay $T$, while having the same ICI of $M_{A}$ streams as the GSM [7]-[9]. More explicitly, the $(T \times M)$-element signal matrix is now constructed by $M_{A}=W=M / T$ number of sparse $(T \times T)$-element submatrices as:

$$
\mathbf{S}=\left[\begin{array}{llll}
\widetilde{\mathbf{S}}_{1} & \widetilde{\mathbf{S}}_{2} & \cdots & \widetilde{\mathbf{S}}_{W}
\end{array}\right]=\sum_{w=1}^{W} s^{l_{w}} \mathbf{A}_{\tau_{w}, \bar{q}_{w}} .
$$

The $(T \times T)$-element submatrices $\left\{\widetilde{\mathbf{S}}_{w}=s^{l_{w}} \widetilde{\mathbf{A}}_{\tau_{w}, \bar{q}_{w}}\right\}_{w=1}^{W}$ are given by the TAST of (13), where $\widetilde{\mathbf{A}}_{\tau_{w}, \bar{q}_{w}}$ is the $(T \times$ $T$ )-element TAST dispersion matrix defined in (13). The holistic dispersion matrix in (19) is given by $\mathbf{A}_{\tau_{w}, \bar{q}_{w}}=$ $\left[\begin{array}{llllllll}\mathbf{0} & \cdots & \mathbf{0} & \widetilde{\mathbf{A}}_{\tau_{w}, \bar{q}_{w}} & \mathbf{0} & \cdots & \mathbf{0}\end{array}\right]$, where the $w$-th submatrix is activated. Owing to the fact that there is only a single non-zero element on each row and column of $\widetilde{\mathbf{S}}_{w}$, the total number of non-zero signals on each row of (19) is given by $W=M_{A}$, which results in the reduced-RF transmission. The throughput of the CE-GSTSK scheme of (19) is given by $R=\frac{W\left(\log _{2} L+\log _{2} \bar{Q}+\log _{2} T\right)}{T}$.

Although each $\widetilde{\mathbf{S}}_{w}$ is ICI-free, the $W=M_{A}$ submatrices in (19) interfer with each other. As a result, the STSK's $(1 \times Q)$ element signal vector of (6) becomes the following GSTSK form:

$$
\begin{aligned}
& \bar{q}_{1}+\left(\tau_{1}-1\right) \bar{Q} \quad \bar{q}_{2}+\left(\tau_{2}-1\right) \bar{Q} \quad \bar{q}_{W}+\left(\tau_{W}-1\right) \bar{Q} \\
& \overline{\mathbf{S}}=[\underbrace{0 \cdots 0 s^{l_{1}} 0 \cdots 0}_{\bar{Q} T} \underbrace{0 \cdots 0 s^{l_{2}} 0 \cdots 0}_{\bar{Q} T} \cdots \underbrace{0 \cdots 0 s^{l_{W}} 0 \cdots 0}_{\bar{Q} T}] .
\end{aligned}
$$

The total number of dispersion matrices is given by $Q=$ $\bar{Q} T W$. It can be readily seen in (20) that the CE-GSTSK imposes the same IAI $=W=M_{A}$ as the GSM [7]-[9].

Example 8: For the case of $M=4$ and $T=2$ at $R=5.0$, a reduced $W=M_{A}=2$ number of RF chains are activated. The $(2 \times 4)$-element signal matrix of (19) is constructed by $\mathbf{S}=\left[\begin{array}{ll}\widetilde{\mathbf{S}}_{1} & \widetilde{\mathbf{S}}_{2}\end{array}\right]$, where $\left\{\widetilde{\mathbf{S}}_{w}\right\}_{w=1}^{2}$ invokes the $(2 \times 2)$-element TAST of (13) associated with $\bar{Q} L=16$. The TAST parameters are the same as those in Example 5, where candidates (A) and (B) respectively achieve $\left(\Lambda_{p}=\Lambda_{s}=0.2706\right)$ and $\left(\Lambda_{p}, \Lambda_{s}\right)=$ $(0.2579,0.3536)$ for the CE-GSTSK of (19). By contrast, the STBC-SM using square 16QAM [6] has the substantially lower diversity gains of $\left(\Lambda_{p}, \Lambda_{s}\right)=(0.194,0.2236)$.

\section{Performance Results}

Considering that $5 \mathrm{G}$ currently supports a maximum of four and eight transmission layers for the uplink and downlink scenarios [50], in this section, we opt for using up to $M=4$ TAs and up to $N=8$ RAs. Secondly, a low $\Delta f_{\text {LOS }}=f_{d}=0.001$ is assumed, where the near-ideal channel estimation scheme of [49] is feasible. Thirdly, the AoA and AoD generally remain near-constant over a frame of signal reception even in the highmobility aeronautical scenarios [49]. Therefore, for the sake of simplificity, we assume $\phi_{r}=\phi_{t}=90^{\circ}$, where the LoSinduced antenna correlation reaches its maximum. Fourthly, without loss of generality, the performance results are presented in both Ricean and Rayleigh scenarios. Finally, for any variable-envelope and non-orthogonal MIMO schemes, their non-zero dB PAPR values and non-zero ICIs are explicitly labelled in all the performance figures. 


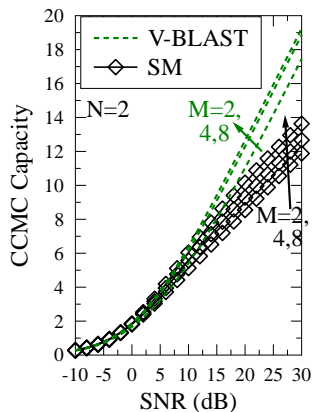

(a) $\mathrm{SM}$

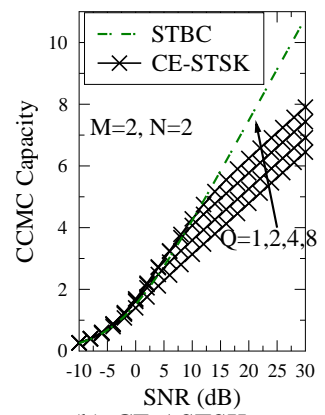

(b) CE-ASTSK
Fig. 6: CCMC capacity of the single-RF SM and CE-ASTSK in Ricean fading $K=0 \mathrm{~dB}$.

\section{A. Capacity and Complexity Comparison}

According to the STSK model of (5), the Continuous-input Continuous-output Memoryless Channel (CCMC) capacity is given by [42]:

$$
\begin{aligned}
C_{S T S K}^{C C M C}(S N R) & =\max _{p(\overline{\mathbf{S}})} \frac{1}{T} \mathcal{I}(\overline{\mathbf{S}} ; \overline{\mathbf{Y}}) \\
& =\max _{p\left(s^{l}\right)} \frac{1}{T} \mathcal{I}\left(s^{l} ; \overline{\mathbf{Y}} \mid q\right)+\max _{p(q)} \frac{1}{T} \mathcal{I}(q ; \overline{\mathbf{Y}}) .
\end{aligned}
$$

The first part in (21) is evaluated based on $p(\overline{\mathbf{Y}} \mid \overline{\mathbf{S}})=$ $\frac{1}{\left(\pi N_{0}\right)^{T N}} \exp \left(-\frac{\|\overline{\mathbf{Y}}-\overline{\mathbf{S H}}\|^{2}}{N_{0}}\right)$ [43] as (22), which is maximized for using the Gaussian Probability Density Function (PDF) for $p\left(s^{l}\right)$, while $\overline{\boldsymbol{\chi}}_{q,-}$ denotes the $q$-th row in $\overline{\boldsymbol{\chi}}$. By contrast, the IM index $q$ is discrete-valued. Therefore, the second part in (21) is evaluated based on $p(\overline{\mathbf{Y}} \mid q)=\frac{1}{\operatorname{det}\left(\pi \mathbf{R}_{Y Y}\right)} \exp \left(-\overline{\mathbf{Y}} \mathbf{R}_{Y Y}^{-1} \overline{\mathbf{Y}}\right)$ relying on $\mathbf{R}_{Y Y}=E\left(\overline{\mathbf{Y}}^{H} \overline{\mathbf{Y}}\right)$ [43] as (23), which is maximized for equiprobable $\left\{p(q)=\frac{1}{Q}\right\}_{q=1}^{Q}$, while $\overline{\mathbf{H}}_{q,-}$ denotes the $q$-th row in $\overline{\mathbf{H}}$ and we have $\left\{\kappa_{q}^{2}=\left\|\overline{\mathbf{H}}_{q,-}\right\|^{2}\right\}_{q=1}^{Q}$. We note that (21)(23) become the CCMC capacity for SM in [67], when we set $Q=M, T=1$ and $\bar{\chi}=\mathbf{I}_{M}$.

The CCMC capacities of SM and CE-ASTSK are portrayed in Fig. 6. In contrast to SM, the spectral efficiency of CEASTSK is improved by increasing $Q$ in Fig. 6(b), which does not require any extra TAs in Fig. 6(a). However, as expected, the CCMC capacities of SM and CE-ASTSK cannot compete with V-BLAST and STBC, respectively. Moreover, the CCMC capacity of CE-ASTSK seen in Fig. 6(b) is lower than that of SM observed in Fig. 6(a), because the mutual information of CE-ASTSK in (21) has to be normalized by the transmission duration of $T$.

However, in reality, the RF chains using real-life PAs always deal with discrete signals, rather than with the 'Gaussianized' signals of (22). The associated Discrete-input Continuousoutput Memoryless Channel (DCMC) capacity evaluated based on $\left[p(\mathbf{Y} \mid \mathbf{S})=\frac{1}{\left(\pi N_{0}\right)^{N T}} \exp \left(-\frac{\|\mathbf{Y}-\mathbf{S H}\|^{2}}{N_{0}}\right)\right]$ [42] is (24), where $\mathcal{I}(\overline{\mathbf{S}} ; \overline{\mathbf{Y}})$ in (21) is maximized for equiprobable $\left\{p\left(\mathbf{S}^{i}\right)=\frac{1}{I}\right\}_{i=0}^{I-1}$. We note that (24) is applicable to all MIMO schemes.

The DCMC capacities of the single-/full-RF schemes are presented in Fig. 7. First of all, Fig. 7(a) demonstrates that SM does not perform well in Ricean fading and the increased Ricean $K$ further deteriorates the SM performance in Fig. 7(b). By contrast, given the same single-RF setup, the proposed CEASTSK is capable of substantially outperforming SM in the face of the increasing Ricean $K$, as evidenced by Figs. 7(a) and 7(b). Furthermore, Fig. 7(c) demonstrates that as a full-RF scheme, the proposed CE-STSK is capable of outperforming

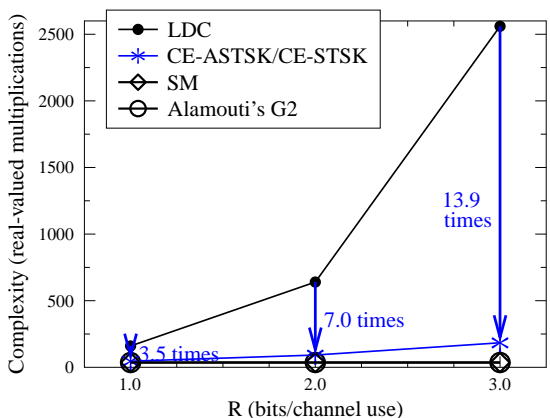

Fig. 8: Complexity comparison between SM, CE-ASTSK/CE-STSK, Alamouti's G2 using $M=2$ and $N=2$ seen in Fig. 7(c) and their LDC counterparts.

Alamouti's G2 STBC, especially as the throughput $R$ and/or the number of RAs $N$ increase.

Lastly, the complexities of the ICI-free MIMO schemes used in Fig. 7 are compared in Fig. 8 in terms of the number of real-valued multiplications [43]. The complexities evaluted for LDC, SM and Alamouti's G2 STBC are $(4 M N+2 N) L^{M}$, $(4 N+7) M$ and $(16 N+4)$, respectively, while that of STSK is equivalent to SM associated with $Q$ TAs and $N T$ RAs. Fig. 8 confirms that all the three ICI-free MIMO schemes exhibit a low detection complexity that does not grow with modulation alphabet size $L$, but the STSK complexity grows linearly with $Q$. Nonetheless, compared to LDC, STSK achieves substantial complexity reductions, as evidenced by Fig. 8 .

\section{B. Two Transmit Antennas}

The proposed CE-ASTSK and CE-STSK are compared to their single-RF and full-RF counterparts in Fig. 9. First of all, it is demonstrated by Fig. 9(a)-(c) that the proposed CEASTSK and CE-STSK outperform the conventional ASTSK and STSK solutions that generate high-PAPR randomized signals [20]-[22]. This verifies the efficiency of our CE design of PAPR $=0 \mathrm{~dB}$ using our improved optimization methodologies. Secondly, in the single-RF mode, Fig. 9(a) demonstrates that the proposed CE-ASTSK substantially outperforms SM. Thirdly, Figs. 9(b)-(c) show that in the full-RF setup, the proposed CE-STSK is also capable of outperforming Alamouti's G2 scheme, especially as $R$ and $N$ increase.

The proposed CE-ASTSK and SM are further compared for a higher throughput $R$ and for higher Ricean $K$ factors in Fig. 10. First of all, SM performs even worse than the single-TA PSK at $R=2.0$ and $K=0 \mathrm{~dB}$, as demonstrated in Fig. 10(a). The SM's performance moderately improves for $R=3.0$ in Fig. 10(b), but degrades in the face of the increased $K=6 \mathrm{~dB}$ value in Fig. 10(c). By contrast, the proposed CEASTSK always substantially outperforms both SM and singleTA PSK, as evidenced in Fig. 10(a)-(c).

\section{Four Transmit Antennas}

In the single-RF mode, Fig. 11 demonstrates that the proposed CE-ASTSK and CE-ASTSK-DR outperform SM-CIOD using high-PAPR signals [23], which once again advocates our CE design. Furthermore, the proposed CE-ASTSK and CEASTSK-DR also achieve substantial diversity gains over SSK and SM, as explicitly marked in Fig. 11. 


$$
\begin{gathered}
C_{S T S K, 1}^{C C M C}(S N R)=\max _{p\left(s^{l}\right)} \frac{1}{T} \mathcal{I}\left(s^{l} ; \overline{\mathbf{Y}} \mid q\right)=\frac{1}{T Q} \sum_{q=1}^{Q} \log _{2}\left(1+\frac{1}{N_{0}}\left\|\overline{\boldsymbol{\chi}}_{q,-} \overline{\mathbf{H}}\right\|^{2}\right), \\
C_{S T S K, 2}^{C C M C}(S N R)=\max _{p(q)} \frac{1}{T} \mathcal{I}(q ; \overline{\mathbf{Y}})=\frac{1}{T Q} \sum_{q=1}^{Q} E\left\{\log _{2} \frac{\frac{Q N_{0}}{N_{0}+\kappa_{q}^{2}} \exp \left[-\overline{\mathbf{Y}}\left(\overline{\mathbf{H}}_{q,-}^{H} \overline{\mathbf{H}}_{q,-}+N_{0} \mathbf{I}_{N T}\right)^{-1} \overline{\mathbf{Y}}^{H}\right]}{\sum_{\bar{q}=1}^{Q} \frac{N_{0}}{N_{0}+\kappa_{\bar{q}}^{2}} \exp \left[-\overline{\mathbf{Y}}\left(\overline{\mathbf{H}}_{\bar{q},-}^{H} \overline{\mathbf{H}}_{\bar{q},-}+N_{0} \mathbf{I}_{N T}\right)^{-1} \overline{\mathbf{Y}}^{H}\right]},\right. \\
\mathcal{C}^{\mathrm{DCMC}}(\mathrm{SNR})=\max _{p(\mathbf{S})} \frac{1}{I T} \sum_{i=0}^{I-1} \int p\left(\mathbf{Y} \mid \mathbf{S}^{i}\right) \log _{2} \frac{I p\left(\mathbf{Y} \mid \mathbf{S}^{i}\right)}{\sum_{i^{\prime}=0}^{I-1} p\left(\mathbf{Y} \mid \mathbf{S}^{i^{\prime}}\right)} d \mathbf{Y}=\frac{1}{I T} \sum_{i=0}^{I-1} \mathrm{E}\left[\log _{2} \frac{I \exp \left(-\frac{\left\|\mathbf{Y}-\mathbf{S}^{i} \mathbf{H}\right\|^{2}}{N_{0}}\right)}{\sum_{i^{\prime}=0}^{I-1} \exp \left(-\frac{\left\|\mathbf{Y}-\mathbf{S}^{i^{\prime}} \mathbf{H}\right\|^{2}}{N_{0}}\right)}\right],
\end{gathered}
$$

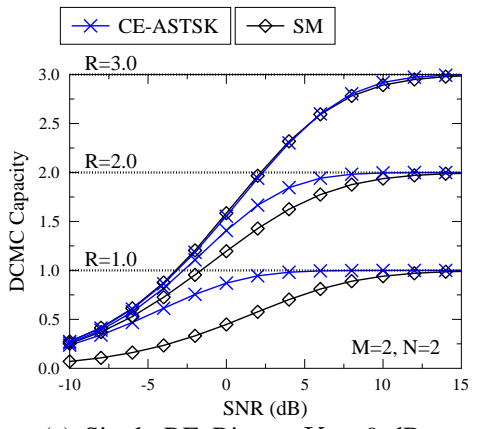

(a) Single-RF, Ricean $K=0 \mathrm{~dB}$

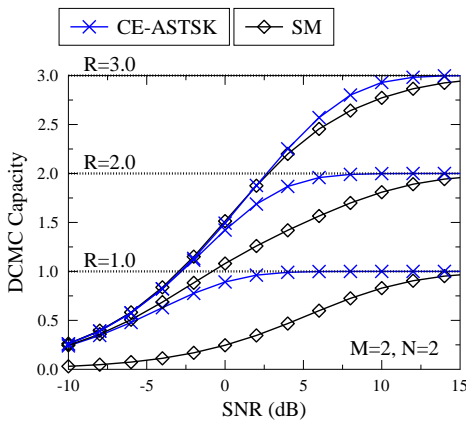

(b) Single-RF, Ricean $K=6 \mathrm{~dB}$

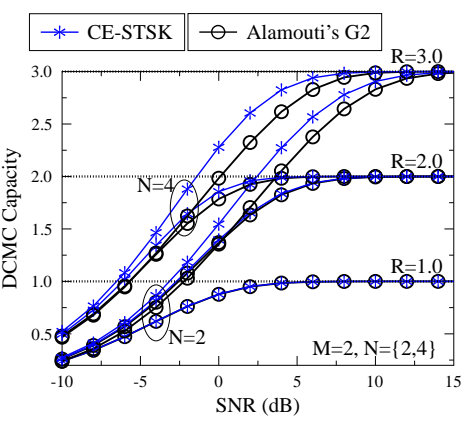

(c) Full-RF, Ricean $K=0 \mathrm{~dB}$

Fig. 7: DCMC capacity of the single-RF schemes of SM [5] and CE-ASTSK as well as the full-RF schemes of Alamouti's G2 [25] and CE-STSK using $M=2$ in Ricean fading.

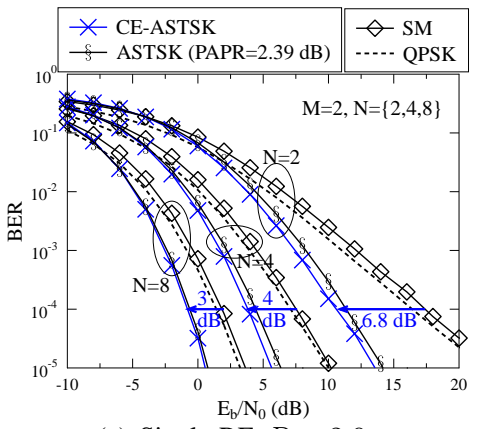

(a) Single-RF, $R=2.0$

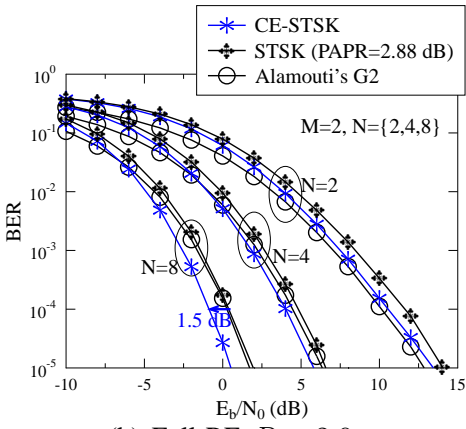

(b) Full-RF, $R=2.0$

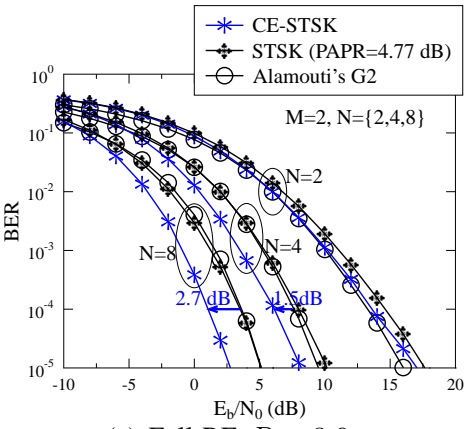

(c) Full-RF, $R=3.0$

Fig. 9: BER performance of the single-RF schemes of SM [5], ASTSK [20], [22] and CE-ASTSK as well as the full-RF schemes of Alamouti's G2 [25], STSK [20], [21] and CE-STSK using $M=2$ in Rayleigh fading.

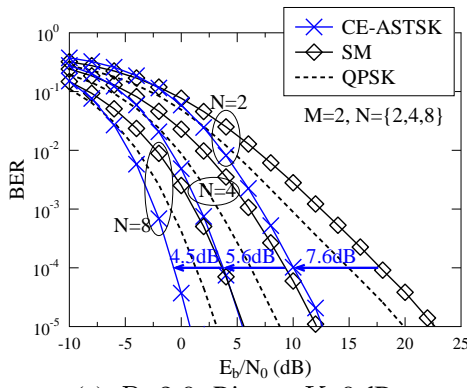

(a) $R=2.0$, Ricean $K=0 \mathrm{~dB}$

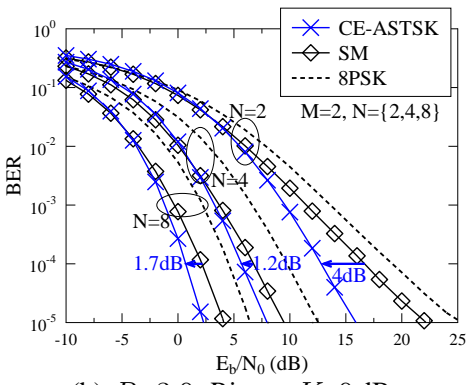

(b) $R=3.0$, Ricean $K=0 \mathrm{~dB}$

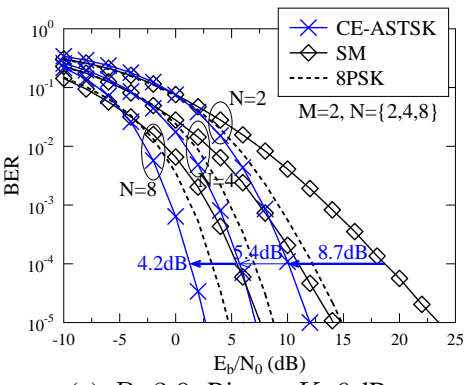

(c) $R=3.0$, Ricean $K=6 \mathrm{~dB}$

Fig. 10: BER performance of the single-RF schemes of SM [5] and CE-ASTSK using $M=2$ in Ricean fading.

In the full-RF mode, Fig. 12 evidences that the proposed CE-STSK and CE-STSK-DR schemes outperform the 1/2-rate and 3/4-rate STBCs [26]-[28] using the same PAPR $=0 \mathrm{~dB}$ of PSK signals. Even after improving the performance of STBCs by using QAM, they still remain substantially inferior to CESTSK and CE-STSK-DR, as seen in Fig. 12.

In the reduced-RF mode, as previously discussed in Sec. IV-C, Fig. 13(a) confirms that the proposed CE-STSK$\mathrm{RH}$ is capable of improving the performance of STBC-SM [6] at low $R=2.0$, but this improvement seems to be less impressive compared to our design in the single-RF and reduced-RF modes. However, as expected, the proposed CE-
GSTSK achieves higher gains in Fig. 13(c) over STBC-SM at $R=5.0$. The price paid is that the proposed CE-GSTSK imposes ICI from two streams, but the STBC-SM resulted in an increased PAPR of $2.55 \mathrm{~dB}$. Moreover, it is unequivocally evidenced by Figs. 13(b) and (d) that the proposed CE-GSTSK substantially outperforms GSM [7]-[9] associated with the same PAPR $=0 \mathrm{~dB}$ and $\mathrm{ICI}=2$.

\section{Turbo Detection Assisted Channel Coded SYSTEM}

Finally, the proposed CE-ASTSK and CE-STSK schemes are amalgamated with LDPC coding, as portrayed in Fig. 14. 


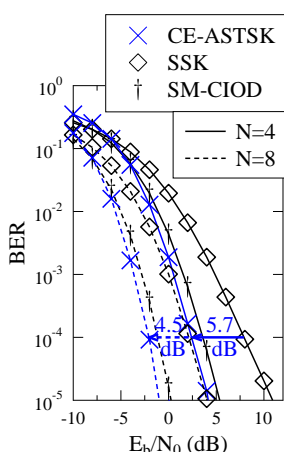

(a) $R=2.0$

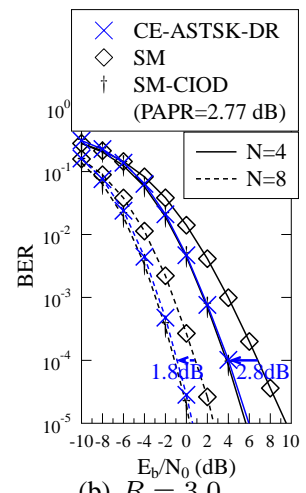

(b) $R=3.0$
Fig. 11: BER performance of the single-RF schemes of SM [5], SM-CIOD [23], CE-ASTSK and CE-ASTSK-DR using $M=4$ in Rayleigh fading.

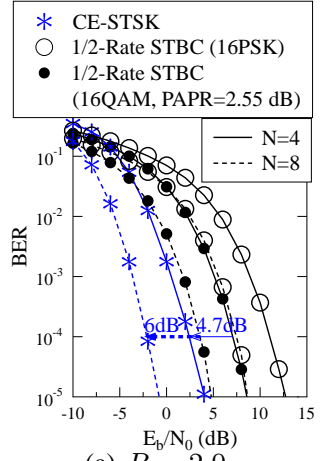

(a) $R=2.0$

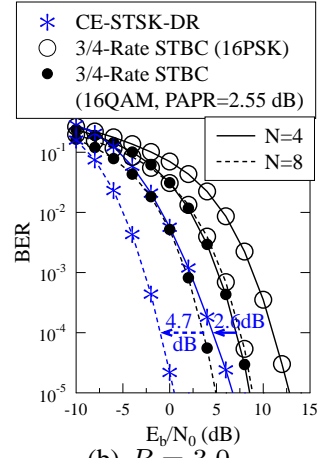

(b) $R=3.0$
Fig. 12: BER performance of the full-RF schemes of 1/2-rate and 3/4rate STBCs [26]-[28], CE-STSK and CE-STSK-DR using $M=4$ in Rayleigh fading.

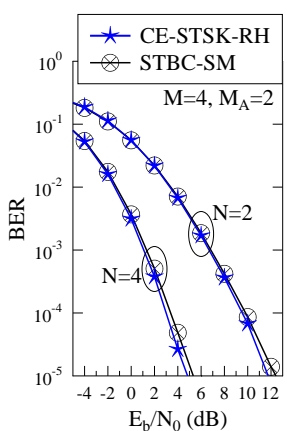

(a) $R=2.0$

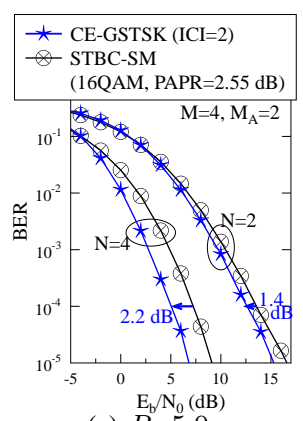

(c) $\stackrel{R}{R=5.0}$

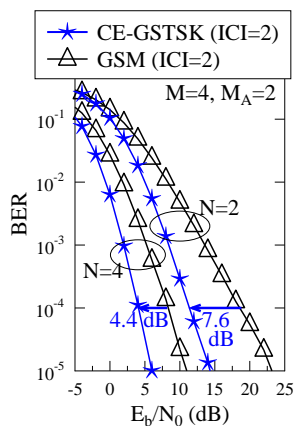

(b) $R=4.0$

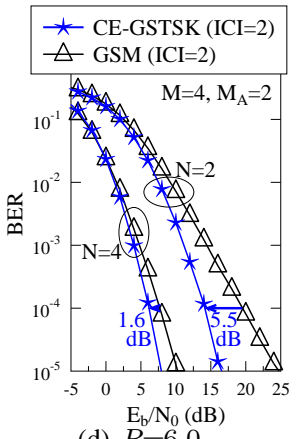

(d) $R=6.0$
Fig. 13: BER performance of the reduced-RF schemes of STBC-SM [6], GSM [7]-[9], CE-STSK-RH and CE-GSTSK using $M=4$ TAs and $M_{A}=2$ activated RFs in Rayleigh fading.
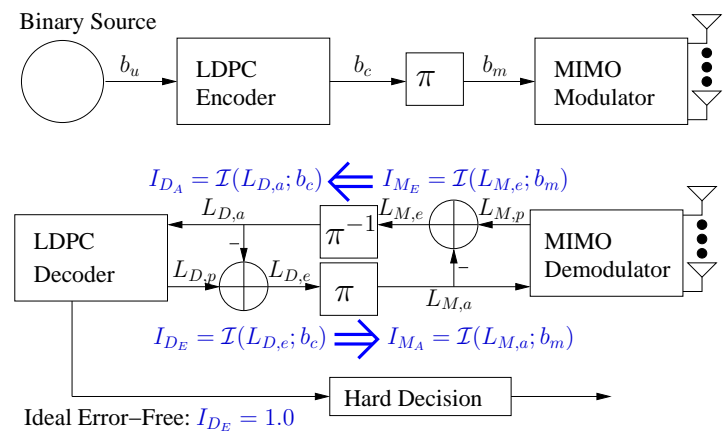

Fig. 14: Schematic of LDPC coded MIMO scheme and its two-stage turbo decoder.

TABLE IV: Configuration of LDPC.

\begin{tabular}{|l|l|}
\hline Description & Configurations \\
\hline \hline Number of neighbors of a variable node & $d_{v}=3$ \\
\hline Number of neighbors of a check node & $d_{c}=6$ \\
\hline Coding rate & 0.5 and 0.8 \\
\hline Number of decoding iterations & 25 \\
\hline Decoding algorithm & Sum-product \\
\hline
\end{tabular}

At the transmitter, the source bits are coded by the LDPC encoder and then interleaved before MIMO mapping. The twostage turbo receiver is constituted by the MIMO demodulator and the LDPC decoder. Both of them produce extrinsic LLRs $L_{e}$, which are obtained by substracting the a priori LLRs $L_{a}$ from the a posteriori LLRs $L_{p}$ formulated as $L_{e}=L_{p}-L_{a}$. The extrinsic LLRs $L_{e}$ are then de-interleaved and fed into the next decoder stage as the a priori LLRs $L_{a}$. In this section, we invoke the reduced-complexity demodulators of [59] for SM and STSK, while the STBCs rely on the reduced-complexity detector of [68]. Furthermore, the $(3,6)$-regular LDPC code is configured according to Table IV, where the coding frame length is set to 10000 bits. The number of LDPC decoding iterations is given by $I R_{L D P C}=25$ in Table IV. The number of outer iterations between the MIMO demodulator and the LDPC decoder in Fig. 14 is set to $I R_{L D P C-M I M O}=4$. For further details on the LDPC decoders, the interested readers may refer to [69].

The EXIT charts [40]-[43] seen in Fig. 15 are invoked for visualizing the convergence behaviour of Fig. 14. The attained mutual information is evaluated from:

$$
\mathcal{I}(b ; L)=\frac{1}{2} \sum_{b=1,0} \int_{-\infty}^{\infty} p(L \mid b) \log _{2} \frac{2 p(L \mid b)}{\sum_{b^{\prime}=1,0} p\left(L \mid b^{\prime}\right)} d L,
$$

where an equiprobable source having $p(b=1)=p(b=0)=$ $\frac{1}{2}$ is assumed, while the PDFs $p(L \mid b=1)$ and $p(L \mid b=0)$ are evaluated by the histograms of $L$ [42], [43]. In Fig. 14, the extrinsic information produced by the MIMO demodulator becomes the a priori information of the LDPC decoder $\mathcal{I}_{M_{E}}=\mathcal{I}_{D_{A}}$. Similarly, the extrinsic information produced by the LDPC decoder becomes the a priori information of the MIMO demodulator $\mathcal{I}_{D_{E}}=\mathcal{I}_{M_{A}}$. When the full knowledge of $\mathcal{I}_{D_{E}}=1.0$ is recovered by the two-stage turbo decoder of Fig. 14, a vanishingly low BER becomes achievable.

More explicitly, the area property of EXIT charts [41][43] quantifies the relationship between the mutual information of (25) and the DCMC capacity of (24) as $A_{M}(\mathrm{SNR})=$ $\int_{0}^{1} \mathcal{I}_{M_{E}} d \mathcal{I}_{M_{A}} \approx \frac{\mathcal{C}^{\mathrm{DCMC}}(\mathrm{SNR})}{\log _{2} I}$. Similarly, the area property for 


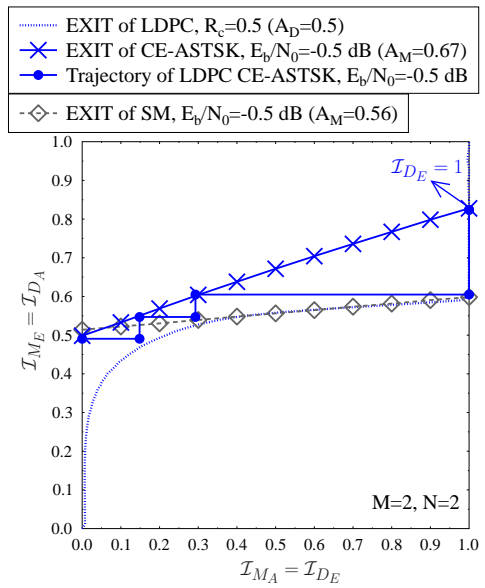

(a) LDPC $R_{c}=0.5$, Ricean $K=0 \mathrm{~dB}$

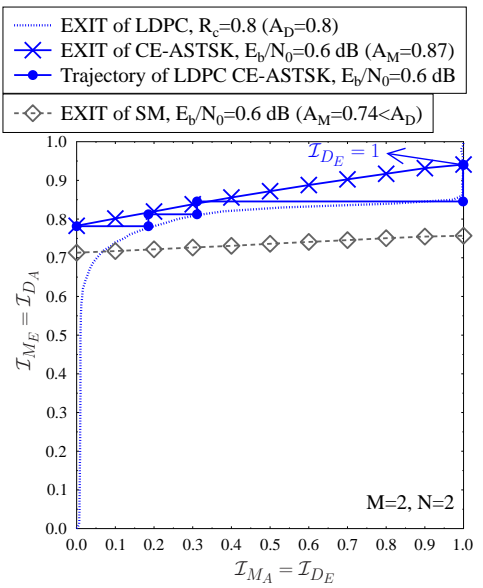

(b) LDPC $R_{c}=0.8$, Ricean $K=0 \mathrm{~dB}$

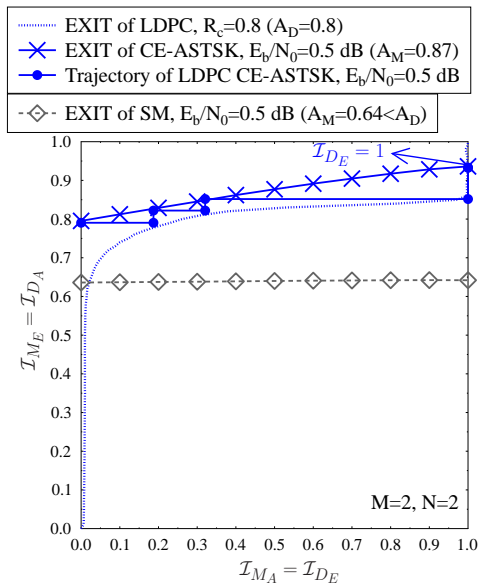

(c) LDPC $R_{c}=0.8$, Ricean $K=6 \mathrm{~dB}$

Fig. 15: EXIT charts and decoding trajectories of LDPC coded single-RF schemes of SM and CE-ASTSK using $M=2$ TAs and $N=2$ RAs at $R=2.0$ in Ricean fading.

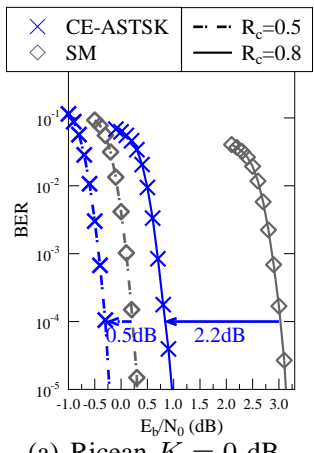

(a) Ricean $K=0 \mathrm{~dB}$

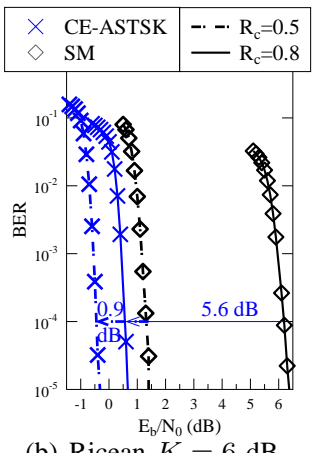

(b) Ricean $K=6 \mathrm{~dB}$

Fig. 16: BER performance of LDPC coded single-RF schemes of SM and CE-ASTSK using $M=2$ TAs and $N=2$ RAs at $R=2.0$ in Ricean fading.

the channel coding results in ${ }^{1} A_{D}=R_{c}$, which is independent of SNR. As a result, in order to achieve decoding convergence for a vanishingly low BER as seen in Fig. 15, the following two requirements have to be satisfied: (I) the area under the EXIT curve of the inner LDPC decoder is higher than that of the outer MIMO demodulator as $A_{M}>A_{D}$; (II) the only intersection point between the EXIT curves of the LDPC decoder and the MIMO demodulator should reach $\mathcal{I}_{D_{E}}=1.0$.

In summary, Fig. 15(a) demonstrates that the soft-decision CE-ASTSK demodulator produces higher extrinsic information than its SM counterpart, where the CE-ASTSK scheme achieves a higher $A_{M}=0.67$ than the SM scheme of $A_{M}=$ 0.56 given the same $E_{b} / N_{0}=0.5 \mathrm{~dB}$. The performance advantages of CE-ASTSK over SM are further increased at higher $E_{b} / N_{0}=0.6 \mathrm{~dB}$ and for a higher Ricean factor of $K=6 \mathrm{~dB}$, as seen in Figs. 15(b) and 15(c), respectively. Moreover, as expected, the decoding trajectories for the LDPC CE-ASTSK arrangement reach $\mathcal{I}_{D_{E}}=1.0$ seen in Figs. 15(a)(c).

Our EXIT chart observations based on Fig. 15 are verified by the BER results of Fig. 16, which demonstrates that the CE-ASTSK schemes outperform their SM counterparts

\footnotetext{
${ }^{1}$ We note that the area property for channel coding was originally evaluated as $\bar{A}_{D}=\int_{0}^{1} \mathcal{I}_{D_{E}} d \mathcal{I}_{D_{A}}=1-R_{c}$ in [41]. The EXIT curves of the LDPC are reversed in Fig. 15 in order to match the inner and outer decoders according to $\mathcal{I}_{M_{E}}=\mathcal{I}_{D_{A}}$ and $\mathcal{I}_{D_{E}}=\mathcal{I}_{M_{A}}$. As a result, we have $A_{D}=1-\bar{A}_{D}=R_{c}$ as seen in Fig. 15.
}

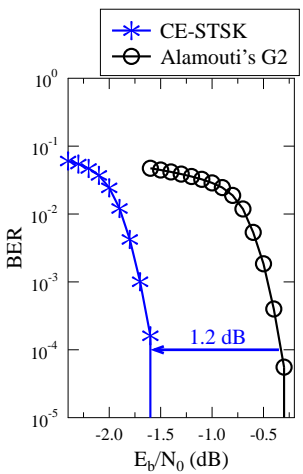

(a) $M=2$

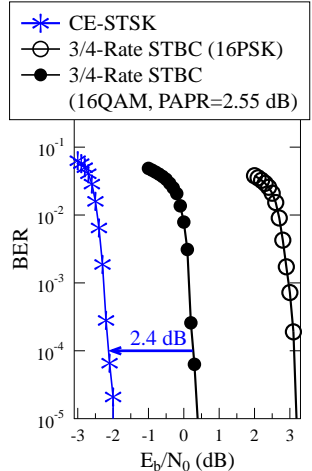

(b) $M=4$
Fig. 17: BER performance of LDPC $\left(R_{c}=0.8\right)$ coded full-RF schemes of Alamouti's G2, 3/4-rate STBC and CE-STSK using $M=\{2,4\}$ TAs and $N=4$ RAs at $R=3.0$ in Ricean fading $(K=0 \mathrm{~dB})$.

in LDPC coded systems. The performance gap observed is further increased for higher LDPC coding rate $R_{c}=0.8$ and for higher Ricean factor $K=6 \mathrm{~dB}$.

The BER results of the LDPC coded full-RF schemes are compared in Fig. 17. First of all, Fig. 17(a) evidences that the CE-STSK arrangement outperforms Alamouti's G2. Secondly, Fig. 17(b) further demonstrates that the CE-STSK arrangement substantially outperforms its 3/4-rate STBC counterpart using PSK associated with the same PAPR $=0 \mathrm{~dB}$. Even when high-PAPR QAM is employed for the 3/4-rate STBC, the CESTSK still performs substantially better in the LDPC coded system, as evidenced by Fig. 17(b).

Lastly, the parameters of the CE-ASTSK/CE-STSK schemes used in Secs. V and VI are summarized in Table V. We note that these parameters are all obtained from our 'offline' optimization methodologies. Our designs are convenient, since the MIMO transceivers only have to store the integer parameters instead of the full signalling matrices.

\section{CONClusions And Future Work}

In conclusion, we have proposed a new family of singleRF CE-ASTSK schemes, which retain the three-fold benefits of PAPR $=0 \mathrm{~dB}$ for signal transmission, ICI-free signal detection and maximized diversity gains. The proposed CEASTSK schemes are shown to substantially outperform their 
TABLE V: Parameters $\left(M, N, T, \bar{Q}, L, L_{D M}, \mathbf{u}, L_{r}, \mathbf{v}\right)$ of the CEASTSK/CE-STSK used in Secs. V and VI.

(a) CE-ASTSK/CE-ASTSK, TAST

$(2,2,2,1,2,2,[1,1], 4,[0,1]):$ Fig.7(c).
$(2,2,2,2,1,2,[1,1], 4,[0,1]):$ Figs.7(a),7(b).
$(2,2,2,2,4,7,[1,6], 8,[0,1]):$ Fig.7(c).
$(2,\{2,4,8\}, 2,4,2,8,[1,3], 8,[0,1]):$ Figs.7(a),7(b),7(c),9(a),9(b),10(a),15,16.
$(2,8,2,16,2,32,[1,7], 16,[0,1]):$ Fig. $10(\mathrm{c})$
$(2,\{4,8\}, 2,4,8,32,[3,27], 64,[0,1]):$ Figs.9(c),10(b),17(a).
$(2,4,2,8,4,32,[1,25], 35,[0,17]):$ Figs.7(c).
$(2,\{2,4\}, 2,4,8,11,[1,10], 16,[0,1]):$ Figs.7(a),7(b),7(c),9(c),10(b),10(c).

(b) CE-ASTSK/CE-ASTSK, PAST

$(4,\{4,8\}, 4,8,2,16,[1,3,5,7], 1024,[0,849,831,650,239,914,308,434$, $684,823,85,986,636,871,422,200])$ : Figs.11(a),12(a).

(c) CE-ASTSK/CE-ASTSK-DR, Type I

$(4,\{4,8\}, 2,8,2,18,[1,5], 12,[0,1]):$ Fig.11(b).
$(4,4,2,2,8,21,[4,17], 25,[0,11]:$ Figs.12(b),17.

$(4,8,2,8,2,18,[1,5], 12,[0,1]:$ Fig. 12(b).

(d) CE-STSK-RH, Type I

$(4,2,2,2,2,4,[1,3], 4,[0,1])$ : Fig.13(a).

$(4,4,2,2,2,4,[1,3], 4,[0,1]):$ Fig.13(a).

(e) CE-GSTSK

$(4,\{2,4\}, 2,4,2,8,[1,3], 8,[0,1]):$ Fig.13(b).
$(4,2,2,2,8,21,[4,17], 25,[0,11]:$ Fig.13(c).
$(4,2,2,4,8,11,[1,10], 16,[0,1]):$ Fig.13(d).
$(4,4,2,8,2,18,[1,5], 12,[0,1]:$ Fig.13(c).
$(4,4,2,8,4,32,[1,25], 35,[0,17]):$ Fig.13(d).

conventional SM counterparts, especially in Ricean fading associated with increasing LoS power. Secondly, we propose the full-RF CE-STSK schemes, which are obtrained upon multiplying the sparse signal matrix by a Hadamard matrix. As a benefit, in the full-RF mode, the proposed CE-STSK schemes become capable of outperforming their STBC counterparts using a large number of RAs without either increasing PAPR or imposing ICI. Thirdly, we have also conceived the reducedRF versions of CE-STSK and CE-GSTSK, which are capable of outperforming their STBC-SM and GSM counterparts. Finally, the proposed schemes are examined in LDPC coded systems, which further confirm the impressive advantages of CE-ASTSK and CE-STSK over SM and STBC in the single$\mathrm{RF}$ and full-RF modes, respectively.

The proposed family of STSK schemes are unambiguously specified by their integer parameters. As a result, the optimization methodology devised in this work invokes bruteforce exhaustive search over the finite integer range for global maxima. We note that the optimization OFs are simplified diversity gains rather than the holistic BER and capacity expressions. The corresponding optimization based on closedform BER and capacity expressions constitutes a compelling future study.

\section{DEDICATION}

Chao $\mathrm{Xu}$ would like to dedicate this paper to the fond memory of Sirui Hu (1985 - 2019), who was an outstanding scientist and an inspiration in the conception of this work.

\section{REFERENCES}

[1] M. D. Renzo, H. Haas, A. Ghrayeb, S. Sugiura, and L. Hanzo, "Spatial modulation for generalized MIMO: Challenges, opportunities, and implementation," Proceedings of the IEEE, vol. 102, pp. 56-103, Jan 2014.

[2] S. Sugiura and L. Hanzo, "Single-RF spatial modulation requires singlecarrier transmission: Frequency-domain turbo equalization for dispersive channels," IEEE Trans. Veh. Technol., vol. 64, pp. 4870-4875, Oct 2015.
[3] P. Yang, Y. Xiao, Y. L. Guan, K. V. S. Hari, A. Chockalingam, S. Sugiura, H. Haas, M. D. Renzo, C. Masouros, Z. Liu, L. Xiao, S. Li, and L. Hanzo, "Single-carrier SM-MIMO: A promising design for broadband large-scale antenna systems," IEEE Commun. Surveys Tutorials, vol. 18, pp. 1687-1716, thirdquarter 2016.

[4] S. Sugiura, T. Ishihara, and M. Nakao, "State-of-the-art design of index modulation in the space, time, and frequency domains: Benefits and fundamental limitations," IEEE Access, vol. 5, pp. 21774-21790, 2017.

[5] R. Y. Mesleh, H. Haas, S. Sinanovic, C. W. Ahn, and S. Yun, "Spatial modulation," IEEE Trans. Veh. Technol., vol. 57, pp. 2228-2241, 2008.

[6] E. Basar, U. Aygolu, E. Panayirci, and H. V. Poor, "Space-time block coded spatial modulation," IEEE Trans. Commun., pp. 823-832, 2011.

[7] J. Jeganathan, A. Ghrayeb, and L. Szczecinski, "Generalized space shift keying modulation for MIMO channels," in IEEE 19th Int. Symp. Pers., Indoor Mobile Radio Commun., pp. 1-5, Sept 2008.

[8] J. Wang, S. Jia, and J. Song, "Generalised spatial modulation system with multiple active transmit antennas and low complexity detection scheme," IEEE Trans. Wireless Commun., vol. 11, pp. 1605-1615, April 2012.

[9] Y. Xiao, Z. Yang, L. Dan, P. Yang, L. Yin, and W. Xiang, "Lowcomplexity signal detection for generalized spatial modulation," IEEE Commun. Lett., vol. 18, pp. 403-406, March 2014.

[10] E. Basar, M. Wen, R. Mesleh, M. D. Renzo, Y. Xiao, and H. Haas, "Index modulation techniques for next-generation wireless networks," IEEE Access, vol. 5, pp. 16693-16746, 2017.

[11] F. H. Raab, P. Asbeck, S. Cripps, P. B. Kenington, Z. B. Popovic, N. Pothecary, J. F. Sevic, and N. O. Sokal, "Power amplifiers and transmitters for RF and microwave," IEEE Trans. Microw. Theory Tech., vol. 50, pp. 814-826, March 2002.

[12] J. Joung, C. K. Ho, K. Adachi, and S. Sun, "A survey on poweramplifier-centric techniques for spectrum- and energy-efficient wireless communications," IEEE Commun. Surveys Tus, vol. 17, pp. 315-333, Firstquarter 2015.

[13] J. Joung, C. K. Ho, and S. Sun, "Spectral efficiency and energy efficiency of OFDM systems: Impact of power amplifiers and countermeasures," IEEE J. Sel. Areas Commun., vol. 32, pp. 208-220, February 2014.

[14] H. Ochiai, "An analysis of band-limited communication systems from amplifier efficiency and distortion perspective," IEEE Trans. Commun., vol. 61, pp. 1460-1472, April 2013.

[15] Z. Hasan, H. Boostanimehr, and V. K. Bhargava, "Green cellular networks: A survey, some research issues and challenges," IEEE Commun. Surveys Tuts, vol. 13, pp. 524-540, Fourth 2011.

[16] L. M. Correia, D. Zeller, O. Blume, D. Ferling, Y. Jading, I. Gdor, G. Auer, and L. V. D. Perre, "Challenges and enabling technologies for energy aware mobile radio networks," IEEE Commun. Mag., vol. 48, pp. 66-72, November 2010.

[17] A. Stavridis, S. Sinanovic, M. Di Renzo, and H. Haas, "Energy evaluation of spatial modulation at a multi-antenna base station," in 2013 IEEE 78th Veh. Technol. Conf. (VTC Fall), pp. 1-5, Sep. 2013.

[18] M. A. Jensen, M. D. Rice, and A. L. Anderson, "Aeronautical telemetry using multiple-antenna transmitters," IEEE Trans. Aerosp. Electron. Syst., vol. 43, pp. 262-272, January 2007.

[19] C. Xu, T. Bai, J. Zhang, R. Rajashekar, R. G. Maunder, Z. Wang, and L. Hanzo, "Adaptive coherent/non-coherent spatial modulation aided unmanned aircraft systems," IEEE Wireless Commun. (accepted).

[20] S. Sugiura, S. Chen, and L. Hanzo, "Coherent and differential spacetime shift keying: A dispersion matrix approach," IEEE Trans. Commun., vol. 58, pp. 3219-3230, November 2010.

[21] S. Sugiura, C. Xu, S. X. Ng, and L. Hanzo, "Reduced-complexity coherent versus non-coherent QAM-aided space-time shift keying," IEEE Trans. Commun., vol. 59, pp. 3090-3101, November 2011.

[22] S. Sugiura, "Coherent versus non-coherent reconfigurable antenna aided virtual MIMO systems," IEEE Signal Process. Lett., vol. 21, pp. 390394, April 2014.

[23] R. Rajashekar and K. V. S. Hari, "Modulation diversity for spatial modulation using complex interleaved orthogonal design," in TENCON 2012 IEEE Region 10 Conf., pp. 1-6, Nov 2012.

[24] C. J. Foschini, "Layered space-time architecture for wireless communication in a fading environment when using multiple antennas," Bell Labs. Tech. J., vol. 1, no. 2, pp. 41-59, 1996.

[25] S. M. Alamouti, "A simple transmit diversity technique for wireless communications," IEEE J. Sel. Areas Commun., vol. 16, pp. 1451-1458, Oct. 1998

[26] V. Tarokh, H. Jafarkhani, and A. R. Calderbank, "Space-time block codes from orthogonal designs," IEEE Trans. Inf. Theory, vol. 45, pp. 1456-1467, July 1999. 
[27] G. Ganesan and P. Stoica, "Space-time block codes: a maximum SNR approach," IEEE Trans. Inf. Theory, vol. 47, pp. 1650-1656, May 2001.

[28] G. Ganesan and P. Stoica, "Space-time diversity using orthogonal and amicable orthogonal designs," Wireless Pers. Commun., vol. 18, no. 2, pp. 165-178, 2001.

[29] H. Jafarkhani, "A quasi-orthogonal space-time block code," IEEE Trans. Commun., vol. 49, pp. 1-4, Jan 2001.

[30] W. Su and X. Xia, "Signal constellations for quasi-orthogonal spacetime block codes with full diversity," IEEE Trans. Inf. Theory, vol. 50, pp. 2331-2347, Oct 2004.

[31] R. W. Heath and A. J. Paulraj, "Linear dispersion codes for MIMO systems based on frame theory," IEEE Trans. Signal Process., vol. 50, pp. 2429-2441, Oct 2002.

[32] B. Hassibi and B. M. Hochwald, "High-rate codes that are linear in space and time," IEEE Trans. Inf. Theory, 2002.

[33] H. E. Gamal and M. O. Damen, "Universal space-time coding," IEEE Trans. Inf. Theory, vol. 49, no. 5, 2003.

[34] B. A. Sethuraman, B. S. Rajan, and V. Shashidhar, "Full-diversity, highrate space-time block codes from division algebras," IEEE Trans. Inf. Theory, vol. 49, pp. 2596-2616, Oct 2003.

[35] J. C. Belfiore, G. Rekaya, and E. Viterbo, "The Golden code: a $2 \times 2$ full-rate space-time code with nonvanishing determinants," IEEE Trans. Inf. Theory, vol. 51, pp. 1432-1436, April 2005.

[36] F. Oggier, G. Rekaya, J. C. Belfiore, and E. Viterbo, "Perfect spacetime block codes," IEEE Trans. Inf. Theory, vol. 52, pp. 3885-3902, Sept 2006

[37] K. P. Srinath and B. S. Rajan, "Improved perfect space-time block codes," IEEE Trans. Inf. Theory, vol. 59, no. 12, 2013.

[38] X. Li and L. Wang, "High rate space-time block coded spatial modulation with cyclic structure," IEEE Commun. Lett., vol. 18, pp. 532-535, April 2014.

[39] M. O. Damen, K. Abed-Meraim, and J. C. Belfiore, "Diagonal algebraic space-time block codes," IEEE Trans. Inf. Theory, pp. 628-636, 2002.

[40] S. ten Brink, "Convergence of iterative decoding," Electron. Lett., vol. 35, pp. 806-808, May 1999.

[41] A. Ashikhmin, G. Kramer, and S. ten Brink, "Extrinsic information transfer functions: model and erasure channel properties," IEEE Trans. Inf. Theory, vol. 50, no. 11, pp. 2657-2673, 2004.

[42] L. Hanzo, O. Alamri, M. El-Hajjar, and N. Wu, Near-Capacity MultiFunctional MIMO Systems: Sphere-Packing, Iterative Detection and Cooperation. John Wiley \& Sons, May 2009.

[43] C. Xu, S. Sugiura, S. X. Ng, P. Zhang, L. Wang, and L. Hanzo, "Two decades of MIMO design tradeoffs and reduced-complexity MIMO detection in near-capacity systems," IEEE Access, vol. 5, pp. 18564 18632, 2017.

[44] C. Xu, P. Zhang, R. Rajashekar, N. Ishikawa, S. Sugiura, L. Wang, and L. Hanzo, "Finite-cardinality single-RF differential space-time modulation for improving the diversity-throughput tradeoff," IEEE Trans. Commun., vol. 67, pp. 318-335, Jan 2019.

[45] C. Xu, P. Zhang, R. Rajashekar, N. Ishikawa, S. Sugiura, Z. Wang, and L. Hanzo, "“Near-perfect" finite-cardinality generalized space-time shift keying," (in preparation).

[46] D. Tse and P. Viswanath, Fundamentals of wireless communication. Cambridge University Press, 2005

[47] S. Sesia, I. Toufik, and M. Baker, LTE - the UMTS long term evolution: from theory to practice. JohnWiley\&Sons, 2011

[48] E. Haas, "Aeronautical channel modeling," IEEE Trans. Veh. Technol., vol. 51, pp. 254-264, Mar 2002.

[49] C. Xu, J. Zhang, T. Bai, P. Botsinis, R. G. Maunder, R. Zhang, and L. Hanzo, "Adaptive coherent/non-coherent single/multiple-antenna aided channel coded ground-to-air aeronautical communication," IEEE Trans. Commun., vol. 67, pp. 1099-1116, Feb 2019.

[50] 3GPP Technical Report 21.915, "Summary of rel-15 work items," [Online]. Available: http://www.3gpp.org/release-15.

[51] 3GPP Technical Specification 38.211, "Nr physical channels and modulation (release 15)," [Online]. Available: http://www.3gpp.org/ftp//Specs/archive/38_series/38.211/.

[52] M. Xiao and T. M. Aulin, "Serially concatenated continuous phase modulation with convolutional codes over rings," IEEE Trans. Commun., vol. 54, pp. 1387-1396, Aug 2006.

[53] Y. Huang, J. Zhang, and M. Xiao, "Constant envelope hybrid precoding for directional millimeter-wave communications," IEEE J. Sel. Areas Commun., vol. 36, pp. 845-859, April 2018.

[54] L. D. Xu, W. He, and S. Li, "Internet of things in industries: A survey," IEEE Trans. Ind. Informat., vol. 10, pp. 2233-2243, Nov 2014
[55] M. R. Palattella, M. Dohler, A. Grieco, G. Rizzo, J. Torsner, T. Engel, and L. Ladid, "Internet of things in the 5G era: Enablers, architecture, and business models," IEEE J. Sel. Areas Commun., vol. 34, pp. 510527, March 2016

[56] K. Song, B. Ji, Y. Huang, M. Xiao, and L. Yang, "Performance analysis of antenna selection in two-way relay networks," IEEE Trans. Signal Process., vol. 63, pp. 2520-2532, May 2015.

[57] P. Yang, Y. Xiao, M. Xiao, and Z. Ma, "NOMA aided precoded spatial modulation for downlink MIMO transmissions," IEEE J. Sel. Topics Signal Process., pp. 1-1, 2019.

[58] R. Sun, D. W. Matolak, and W. Rayess, "Air-ground channel characterization for unmanned aircraft systems - part IV: Airframe shadowing," IEEE Trans. Veh. Technol., vol. 66, pp. 7643-7652, Sept 2017.

[59] C. Xu, S. Sugiura, S. X. Ng, and L. Hanzo, "Spatial modulation and space-time shift keying: Optimal performance at a reduced detection complexity," IEEE Trans. Commun., vol. 61, pp. 206-216, January 2013.

[60] R. Rajashekar, K. V. S. Hari, and L. Hanzo, "Reduced-complexity ML detection and capacity-optimized training for spatial modulation systems," IEEE Trans. Commun., vol. 62, pp. 112-125, January 2014.

[61] A. Goldsmith, Wireless communications. Cambridge University Press, 2005.

[62] X. Liang and X. Xia, "Unitary signal constellations for differential space-time modulation with two transmit antennas: parametric codes, optimal designs, and bounds," IEEE Trans. Inf. Theory, vol. 48, pp. 22912322, Aug 2002.

[63] A. G. Helmy, M. D. Renzo, and N. Al-Dhahir, "Enhanced-reliability cyclic generalized spatial-and-temporal modulation," IEEE Commun. Lett., vol. 20, pp. 2374-2377, Dec 2016.

[64] M. Le, V. Ngo, H. Mai, X. N. Tran, and M. D. Renzo, "Spatially modulated orthogonal space-time block codes with non-vanishing determinants," IEEE Trans. Commun., vol. 62, pp. 85-99, January 2014.

[65] C. Jeon and J. W. Lee, "Multi-strata space-time coded spatial modulation," IEEE Commun. Lett., vol. 19, no. 11, 2015.

[66] P. Maheswaran and M. D. Selvaraj, "Multi-RF chain time successive space-shift-keying-M-ary modulation: A transmit diversity scheme," IEEE Trans. Veh. Technol., vol. 66, pp. 7086-7097, Aug 2017.

[67] Y. Yang and B. Jiao, "Information-guided channel-hopping for high data rate wireless communication," IEEE Commun. Lett., vol. 12, pp. 225227, April 2008.

[68] C. Xu, D. Liang, S. Sugiura, S. X. Ng, and L. Hanzo, "Reducedcomplexity Approx-Log-MAP and Max-Log-MAP soft PSK/QAM detection algorithms," IEEE Trans. Commun., vol. 61, pp. 1415-1425, April 2013.

[69] P. Hailes, L. Xu, R. G. Maunder, B. M. Al-Hashimi, and L. Hanzo, "A survey of FPGA-based LDPC decoders," IEEE Commun. Surveys Tuts., vol. 18, pp. 1098-1122, Secondquarter 2016.

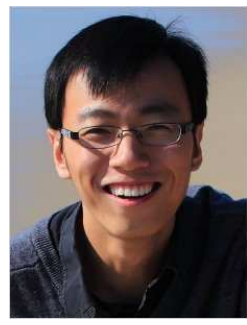

Chao Xu (S'09-M'14) received a B.Eng. degree from Beijing University of Posts and Telecommunications, China, and a BSc(Eng) with First Class Honours from Queen Mary, University of London, UK, through a Sino-UK joint degree program in 2008, both in Telecommunications Enginneering with Management. He obtained a MSc degree with distinction in Radio Frequency Communication Systems and a Ph.D. degree in Wireless Communications from the University of Southampton, UK in 2009 and 2015, respectively. He is currently a postdoctoral researcher working at Next Generation Wireless Research Group, University of Southampton, UK. His research interests include index modulation, reduced-complexity MIMO design, noncoherent detection and turbo detection. He was awarded the Best M.Sc. Student in Broadband and Mobile Communication Networks by the IEEE Communications Society (United Kingdom and Republic of Ireland Chapter) in 2009. He also received 2012 Chinese Government Award for Outstanding Self-Financed Student Abroad. 


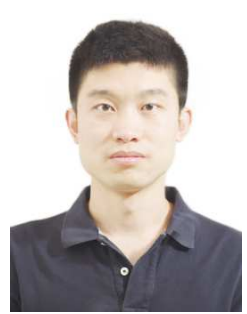

Tong Bai (S'15-M'19) received the B.Sc. degree in telecommunications from Northwestern Polytechnical University, Xi'an, China, in 2013, the M.Sc. and $\mathrm{Ph} . \mathrm{D}$. degrees in communications and signal processing from University of Southampton, U.K. in 2014 and 2019, respectively. Since 2019, he has been a Postdoctoral Researcher in Queen Mary University of London, U.K. His research interests include the performance analysis, transceiver design and utility optimization for power-line and wireless communications.

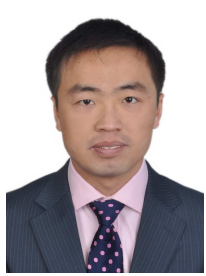

Jiankang Zhang (S'08-M'12-SM'18) received the B.Sc. degree in Mathematics and Applied Mathematics from Beijing University of Posts and Telecommunications in 2006, and the Ph.D. degree in Communication and Information Systems from Zhengzhou University in 2012. Dr Zhang was a lecturer from 2012 to 2013 and an associate professor from 2013 to 2014 in School of Information Engineering, Zhengzhou University. From 2009 to 2011 , Dr Zhang was a visiting PhD student in the School of Electronics and Computer Science, the University of Southampton, UK. Currently, he is a Senior Research Fellow in the University of Southampton, UK. Dr Zhang is a recipient of a number of academic awards, including Excellent Doctoral Dissertation of Henan Province, China, Youth Science and Technology Award of Henan Province, China. His research interests are in the areas of wireless communications and signal processing, aeronautical communications and broadband communications. He serves as an Associate Editor for IEEE ACCESS and a guest editor of a special issue on EURASIP Journal on Advances in Signal Processing.

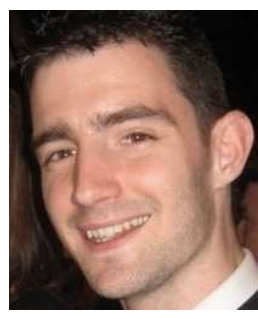

Robert G. Maunder Prof Robert G. Maunder has studied with the School of Electronics and Computer Science, University of Southampton, UK, since October 2000. He was awarded a first class honours BEng in Electronic Engineering in July 2003, as well as a $\mathrm{PhD}$ in Telecommunications in December 2007. He began a lectureship in November 2007 and was promoted to Associate Professor in March 2013 and to Professor in August 2017. He was awarded Senior Member status of the IEEE in December 2012, Chartered Engineer status of the IET in November 2013 and Fellow status of the IET in January 2017. Rob's research interests include joint source/channel coding and the holistic design of algorithms and hardware implementations for wireless communications. He has published around 100 IEEE papers in these areas. He is the founder and CTO of AccelerComm Ltd, which is commercialising his research as soft-IP.

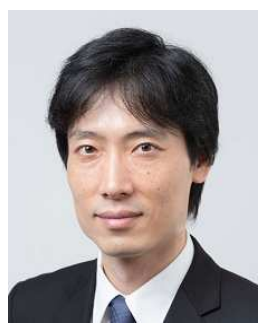

Shinya Sugiura (M'06-SM'12) received the B.S. and M.S. degrees in aeronautics and astronautics from Kyoto University, Kyoto, Japan, in 2002 and 2004 , respectively, and the Ph.D. degree in electronics and electrical engineering from the University of Southampton, Southampton, U.K., in 2010.

From 2004 to 2012, he was a Research Scientist with Toyota Central Research and Development Laboratories, Inc., Aichi, Japan. From 2013 to 2018, he was an Associate Professor with the Department of Computer and Information Sciences, Tokyo University of Agriculture and Technology, Tokyo, Japan. Since 2018, he has been an Associate Professor with the Institute of Industrial Science, The University of Tokyo, Tokyo, Japan, where he heads the Wireless Communications Research Group. His research has covered a range of areas in wireless communications, networking, signal processing, and antenna technology. $\mathrm{He}$ authored or coauthored over 60 IEEE journal papers.

Dr. Sugiura was a recipient of a number of awards, including the Fifth Yasuharu Suematsu Award in 2019, the Sixth RIEC Award from the Foundation for the Promotion of Electrical Communication in 2016, the Young Scientists' Prize by the Minister of Education, Culture, Sports, Science and Technology of Japan in 2016, the 14th Funai Information Technology Award (First Prize) from the Funai Foundation in 2015, the 28th Telecom System Technology Award from the Telecommunications Advancement Foundation in 2013, the Sixth IEEE Communications Society Asia-Pacific Outstanding Young Researcher Award in 2011, the 13th Ericsson Young Scientist Award in 2011, and the 2008 IEEE Antennas and Propagation Society Japan Chapter Young Engineer Award. He was also certified as an Exemplary Reviewer of IEEE COMMUNICATIONS LETTERS in 2013 and 2014, and that of IEEE TRANSACTIONS ON COMMUNICATIONS in 2018.

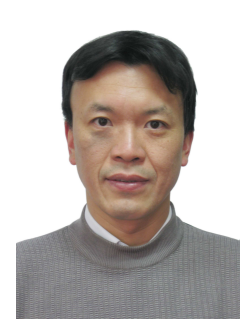

Zhaocheng Wang Prof. Zhaocheng Wang received his B.S., M.S. and Ph.D. degrees from Tsinghua University in 1991, 1993 and 1996, respectively. From 1996 to 1997, he was a Post-Doctoral Fellow with Nanyang Technological University, Singapore. From 1997 to 1999, he was a Research Engineer/Senior Engineer with OKI Techno Centre Pte. Ltd., Singapore. From 1999 to 2009, he was a Senior Engineer/Principal Engineer with Sony Deutschland GmbH, Germany. Since 2009, he has been a Professor with Department of Electronic Engineering, Tsinghua University, where he is currently the Director of Broadband Communication Key Laboratory, Beijing National Research Center for Information Science and Technology (BNRist).

His research interests include wireless communications, millimeter wave communications and optical wireless communications. Prof. Wang published more than 150 peer-reviewed journal papers. He hold 34 US/EU granted patents ( 23 of them as the first inventor). Several granted US/EU patents have been accepted by a plurality of international standards like IEEE802.11n, DVB-T2, IEEE802.15.3c, ECMA387 and etc. Besides that, he authored or co-authored two books, which have been selected by IEEE Series on Digital \& Mobile Communication and published by Wiley-IEEE Press.

Prof. Wang is a Fellow of the Institution of Engineering and Technology. He received ICC2013 Best Paper Award, OECC2015 Best Student Paper Award, 2016 IEEE Scott Helt Memorial Award (Best Paper Award of IEEE Transactions on Broadcasting), 2016 IET Premium Award (Best Paper Award of Electronics Letters), 2016 National Award for Science and Technology Progress (First Prize), ICC2017 Best Paper Award and 2018 IEEE ComSoc Asia-Pacific Outstanding Paper Award. He was an Associate Editor of IEEE Transactions on Wireless Communications from 2011 to 2015 and an Associate Editor of IEEE Communications Letters from 2013 to 2016. He was also Signal Processing for Optical Wireless Communications Symposium CoChair of IEEE GlobalSIP2015, Optical Wireless Communications Symposium Co-Chair of OECC2015 and Wireless Communications Symposium Co-Chair of IEEE ICC 2013. 


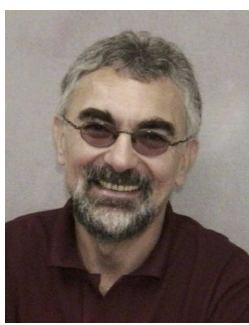

Lajos Hanzo (M'91-SM'92-F'04) Lajos Hanzo (http://www-mobile.ecs.soton.ac.uk) FREng, F'04, FIET, Fellow of EURASIP, received his 5-year degree in electronics in 1976 and his doctorate in 1983 from the Technical University of Budapest. In 2009 he was awarded an honorary doctorate by the Technical University of Budapest and in 2015 by the University of Edinburgh. In 2016 he was admitted to the Hungarian Academy of Science. During his 40-year career in telecommunications he has held various research and academic posts in Hungary, Germany and the UK. Since 1986 he has been with the School of Electronics and Computer Science, University of Southampton, UK, where he holds the chair in telecommunications. He has successfully supervised $119 \mathrm{PhD}$ students, co-authored 18 John Wiley/IEEE Press books on mobile radio communications totalling in excess of 10000 pages, published $1800+$ research contributions at IEEE Xplore, acted both as TPC and General Chair of IEEE conferences, presented keynote lectures and has been awarded a number of distinctions. Currently he is directing a 60-strong academic research team, working on a range of research projects in the field of wireless multimedia communications sponsored by industry, the Engineering and Physical Sciences Research Council (EPSRC) UK, the European Research Council's Advanced Fellow Grant and the Royal Society's Wolfson Research Merit Award. He is an enthusiastic supporter of industrial and academic liaison and he offers a range of industrial courses. He is also a Governor of the IEEE ComSoc and VTS. He is a former Editor-in-Chief of the IEEE Press and a former Chaired Professor also at Tsinghua University, Beijing. For further information on research in progress and associated publications please refer to http://www-mobile.ecs.soton.ac.uk 